\title{
Neuropathic pain in a Fabry disease rat model
}

\author{
James J. Miller, ${ }^{1}$ Kazuhiro Aoki, ${ }^{2}$ Francie Moehring, ${ }^{3}$ Carly A. Murphy, ${ }^{1}$ Crystal L. O'Hara, ${ }^{3}$ \\ Michael Tiemeyer, ${ }^{2}$ Cheryl L. Stucky, ${ }^{3}$ and Nancy M. Dahms ${ }^{1}$ \\ 'Department of Biochemistry, Medical College of Wisconsin, Milwaukee, Wisconsin, USA. ${ }^{2}$ Complex Carbohydrate Research \\ Center, University of Georgia, Athens, Georgia, USA. ${ }^{3}$ Department of Cell Biology, Neurobiology, \& Anatomy, Medical \\ College of Wisconsin, Milwaukee, Wisconsin, USA.
}

Fabry disease, the most common lysosomal storage disease, affects multiple organs and results in a shortened life span. This disease is caused by a deficiency of the lysosomal enzyme $\alpha$-galactosidase A, which leads to glycosphingolipid accumulation in many cell types. Neuropathic pain is an early and severely debilitating symptom in patients with Fabry disease, but the cellular and molecular mechanisms that cause the pain are unknown. We generated a rat model of Fabry disease, the first nonmouse model to our knowledge. Fabry rats had substantial serum and tissue accumulation of $\alpha$-galactosyl glycosphingolipids and had pronounced mechanical pain behavior. Additionally, Fabry rat dorsal root ganglia displayed global $\mathrm{N}$-glycan alterations, sensory neurons were laden with inclusions, and sensory neuron somata exhibited prominent sensitization to mechanical force. We found that the cation channel transient receptor potential ankyrin 1 (TRPA1) is sensitized in Fabry rat sensory neurons and that TRPA1 antagonism reversed the behavioral mechanical sensitization. This study points toward TRPA1 as a potentially novel target to treat the pain experienced by patients with Fabry disease.

Authorship note: JJM, KA, and FM contributed equally to the work.

Conflict of interest: The authors have declared that no conflict of interest exists.

Submitted: December 11, 2017 Accepted: February 7, 2018 Published: March 22, 2018

Reference information: JCI Insight. 2018;3(6):e99171. https:// doi.org/10.1172/ji.insight.99171

\section{Introduction}

Fabry disease (OMIM \#301500) is an X-linked lysosomal storage disease caused by a deficiency of the lysosomal hydrolase, $\alpha$-galactosidase A ( $\alpha$-Gal A, encoded by the GLA gene). Deficient $\alpha$-Gal A activity results in aberrant accumulation of glycosphingolipids (GSLs), including globotriaosylceramide (Gb3) and its metabolite, globotriaosylsphingosine (lyso-Gb3) (Figure 1). The abnormal buildup of GSLs leads to cellular dysfunction that progresses to multisystem organ pathophysiology by incompletely understood mechanisms. Common complications of Fabry disease include corneal and lenticular opacities, dermatological abnormalities (e.g., anhidrosis, angiokeratomas), renal insufficiency, gastrointestinal distress, cardiac malfunction, and cerebrovascular disease (reviewed in refs. 1, 2). These complications lead to a decreased life expectancy, where median survivals of affected male and female patients are 57 and 72 years, respectively (3). One of the most problematic symptoms is episodic and chronic pain that starts in childhood. Fabry patients experience both whole body diffuse pain and distal extremity-focused pain in the hands and feet. The pain can be spontaneous or elicited by skin touch, and patients often have pain crisis attacks triggered by physical exercise, heat exposure, or fever (4). These daily symptoms lead to a substantially compromised quality of life. Pain treatments, such as antiepileptics and opioids, have significant side effects including addiction and do not completely ameliorate the pain experienced by patients. Therefore, novel and targeted pain treatments are greatly needed.

Mouse models of Fabry disease have been developed, but they do not exhibit all of the typical symptoms seen in patients (5-7), and behavioral studies to assess pain show conflicting results (8-10). Given that there are no other animal models of Fabry disease, we asked if $\alpha$-Gal A deficiency in a larger species would result in a phenotype that better recapitulates the neuropathic pain symptoms experienced by patients. We chose the rat to generate an animal model of Fabry disease. Compared with mice, rat neural circuitry pathways have been well characterized, behavior is less variable, and monitoring and manipulation of numerous physiological processes is easier due to their $\sim 10$-fold larger size. All of these attributes in rats are especially advantageous when assessing pain phenotypes and studying the mechanistic basis of pain development. Together with toxicology studies that are well established in rats, we predicted that a Fabry 


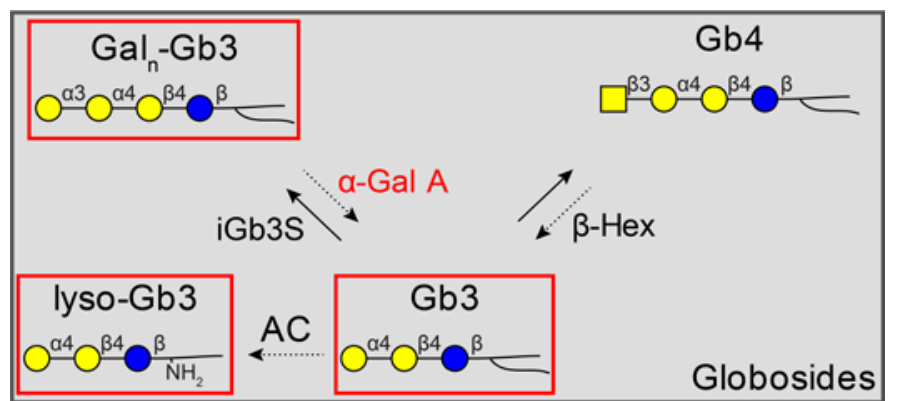

Gb3S $\uparrow$, $a-$ Gal A
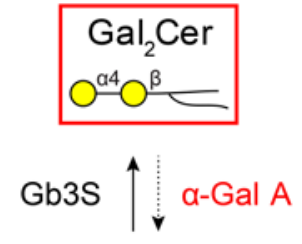

GalCer<smiles>C=C[OH2+]</smiles>

Sphingosine $\overline{\mathrm{NH}_{2}}$

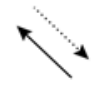
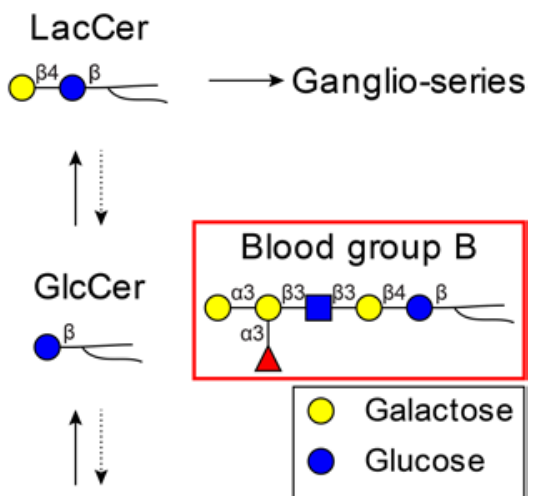

Ceramide
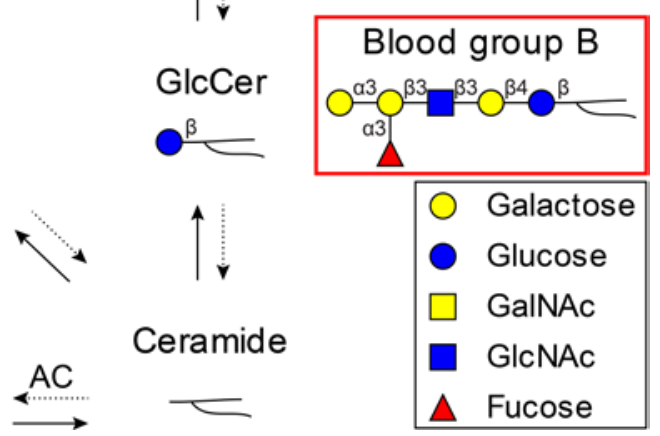

Figure 1. Biosynthesis and degradation of globoside glycosphingolipids (GSLs). GSLs are synthesized (solid arrows) by the sequential addition of monosaccharides to ceramide. Globotriaosylceramide synthase (Gb3S) catalyzes the addition of $\alpha$-1,4-galactose to lactosylceramide (LacCer), which produces globotriaosylceramide (Gb3). Isoglobotriaosylceramide synthase (iGb3S) can add additional $\alpha-1,3$-galactose residues to Gb3, forming polygalactosylated species ( $\mathrm{Gal}_{\mathrm{n}}-\mathrm{Gb} 3$ ). GSL degradation (dashed arrows) occurs in lysosomes by acid hydrolases. $\beta$-Hexosaminidase ( $\beta$-Hex) removes terminal $N$-acetylgalactosamine (GalNAc) from globotetraosylceramide (Gb4). Acid ceramidase (AC) removes the fatty acid chain from $\mathrm{Gb} 3$ to form globotriaosylsphingosine (lyso-Gb3). $\alpha$-Galactosidase $A$ ( $\alpha$-Gal A, red) removes terminal $\alpha$-galactose residues from GSLs. In Fabry disease, $\alpha$-Gal A is deficient, and substrates containing terminal $\alpha$-galactose (e.g., Gb3, lyso-Gb3, galabiosylceramide [Gal, Cer], blood group B GSLs) accumulate. Globoside GSLs are shown in the shaded gray box. GSLs whose abundance increases in Fabry rats are contained within the red boxes. Graphical representations of monosaccharide residues are shown in the boxed legend and are consistent with the symbol nomenclature for glycans. GalCer, galactosylceramide; GlcCer, glucosylceramide; GIcNAc, $\mathrm{N}$-acetylglucosamine.

rat would be a useful new tool in preclinical studies to assess novel treatments, optimize existing therapies, and identify the mechanisms that drive neuropathic pain in Fabry disease and other diseases.

Using CRISPR/Cas9 technology, we generated a Gla-KO rat, the first nonmouse model of Fabry disease to our knowledge. The Fabry rat, which is completely deficient in $\alpha$-Gal A activity, accumulates the established GSL biomarkers, Gb3 and lyso-Gb3, and develops mechanical pain behavior. We observed $\mathrm{N}$-glycan alterations in dorsal root ganglia (DRG) and show that primary sensory neuron somata from Fabry rats are sensitized to mechanical probing. We found that the cation channel transient receptor potential ankyrin 1 (TRPA1) is sensitized in Fabry rat sensory neurons and that the TRPA1 antagonist HC-030031 reverses the mechanical pain behavior in Fabry rats. Therefore, we show that this Fabry rat model allows for the elucidation of disease mechanisms and testing therapies to treat pain symptoms in Fabry disease and may aid in the identification of new therapies for other neuropathic pain syndromes.

\section{Results}

Fabry rat model generation and confirmation of $\alpha$-Gal A deficiency. A Fabry rat model was generated on the Dark Agouti (DA) strain. CRISPR/Cas9 technology was used to disrupt exon 2 of the Gla gene, which encodes $\alpha$-Gal A (Figure 2A). A single male founder was identified, and this mosaic rat had both a 4-bp deletion and a 47-bp deletion in the Gla gene. However, only the 47-bp deletion was transmitted through the germ line. The 47-bp deletion leads to a frameshift, resulting in 13 new amino acids followed by a premature stop codon (Figure 2A). Because this 100-residue truncated protein product does not contain conserved aspartic acid residues essential for catalysis (11), it was predicted to be nonfunctional.

To confirm this prediction, $\alpha$-Gal A activity was assayed in serum and liver homogenates. Unlike WT rats, which have normal $\alpha$-Gal A activity levels, $\alpha$-Gal A activity was undetectable in $\mathrm{KO}$ male and female serum and liver (Figure 2B). $\alpha-G a l$ A activity was detectable in serum and liver of heterozygous (HET) females, but the enzymatic activity was significantly reduced compared with WT females (Figure 2B). As controls, we assayed the activities of 2 other lysosomal glycosidases. Both $\beta$-hexosaminidase (Figure 2C) 

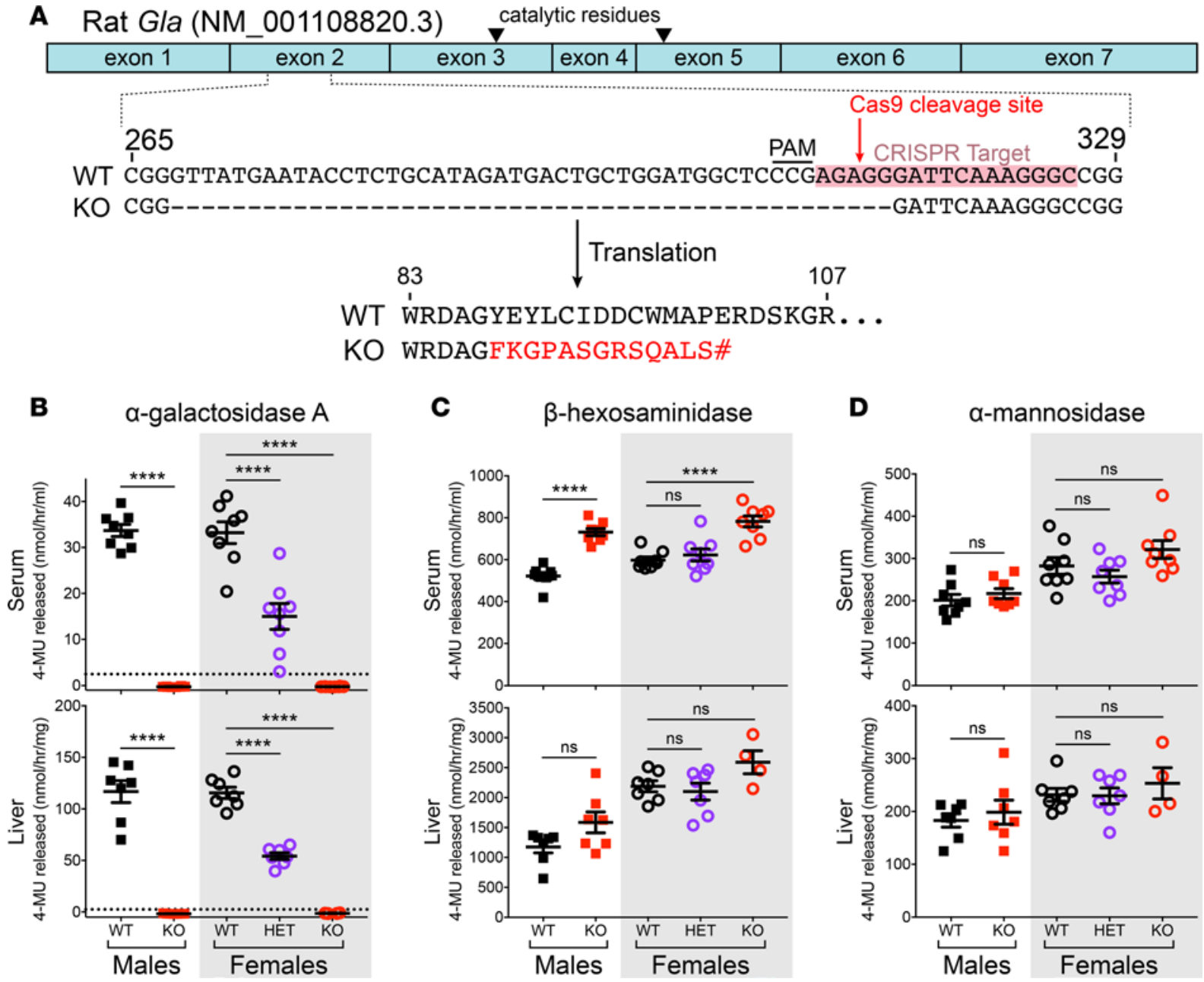

Figure 2. Fabry rat generation and activities of selected lysosomal enzymes. (A) The rat Gla gene encodes $\alpha$-galactosidase $A$ ( $\alpha$ - $G$ al $A$ ), and consists of 7 exons (light blue rectangles). The genomic locations of highly conserved, catalytic residues (Asp172 and Asp233) are identified by the black triangles. A portion of the nucleotide sequence of exon 2 is shown with the CRISPR target highlighted, along with the Cas9 cleavage site. The protospacer adjacent motif (PAM) site is also identified. Translations of the WT (black) and the KO ( -47 bp deletion, red) sequences are also shown with the premature stop codon (\#). (B-D) Lysosomal enzymes were assayed in rat serum and liver using 4-methylumbelliferyl (4-MU) substrates for $\alpha$-Gal A (B), $\beta$-hexosaminidase (C), and $\alpha$-mannosidase (D). Enzyme activity was measured in male (unshaded) and female (shaded) 13-week-old rat tissues, and each symbol represents a biological replicate, each containing 3 technical replicates. Serum biological replicates include $n=8$ for each genotype and sex. Liver biological replicates include $n=7$ for WT males, KO males, WT females, and heterozygous (HET) females and $n=4$ for KO females. The horizontal dashed line in B indicates the threshold of detection. Shown are mean \pm SEM. Male means were compared using an unpaired, 2-tailed $t$ test. Female means were compared using 1 -way ANOVA and Dunnett's multiple comparison test. ${ }^{* * *} P<0.0001$.

and $\alpha$-mannosidase (Figure 2D) - which are involved in GSL and N-glycan metabolism, respectively are expressed in $\mathrm{KO}$ rats. The activity of liver $\beta$-hexosaminidase (Figure $2 \mathrm{C}$ ) and $\alpha$-mannosidase (Figure 2D) did not differ between WT and KO rats, whereas serum $\beta$-hexosaminidase was slightly elevated in KO animals (Figure 2C). Thus, the Gla-KO rats are completely deficient in $\alpha$-Gal A activity, while the activity levels of 2 other lysosomal enzymes remain stable.

Fabry rats accumulate $\alpha$-galactosyl glycosphingolipids in serum and RBC. Patients with Fabry disease accumulate GSLs containing terminal $\alpha$-galactose residues in serum and tissues. These $\alpha$-galactosyl GSLs include Gb3, lyso-Gb3, galabiosylceramide, and blood group B GSLs (Figure 1, red boxes). As $\alpha$-Gal A activity was shown to be undetectable in Gla-KO rats (Figure 2B), we next determined whether these rats accumulated the $\alpha$-Gal A substrates, $\alpha$-galactosyl GSLs, using nanospray ionization-mass spectrometry (NSI-MS). Gb3 was significantly elevated in KO serum compared with WT serum by 100 -fold (Figure 3A). Lyso-Gb3 and blood group B were detected in KO serum at $151 \pm 6 \mathrm{nM}$ and $131 \pm 15 \mathrm{nM}$, respectively (mean \pm SEM); however, these GSL species were undetectable in WT serum (Figure $3 \mathrm{~A}$ ). 


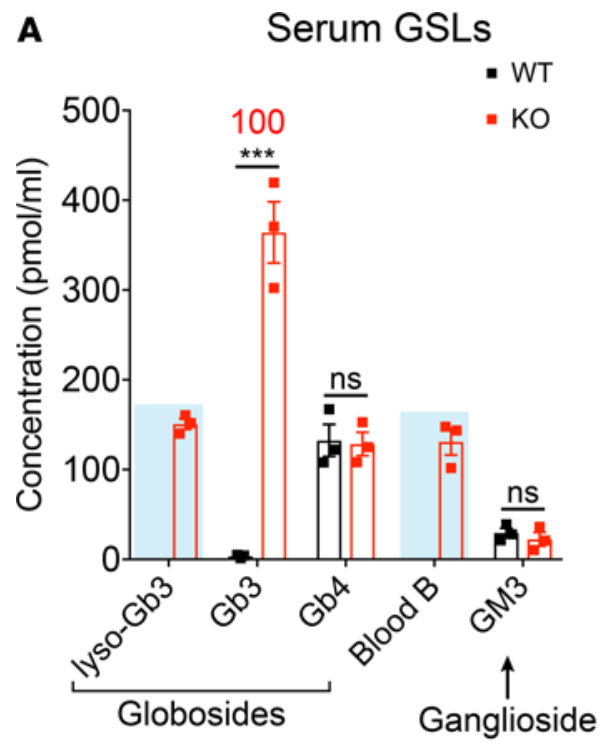

B $\quad$ RBC GSLs

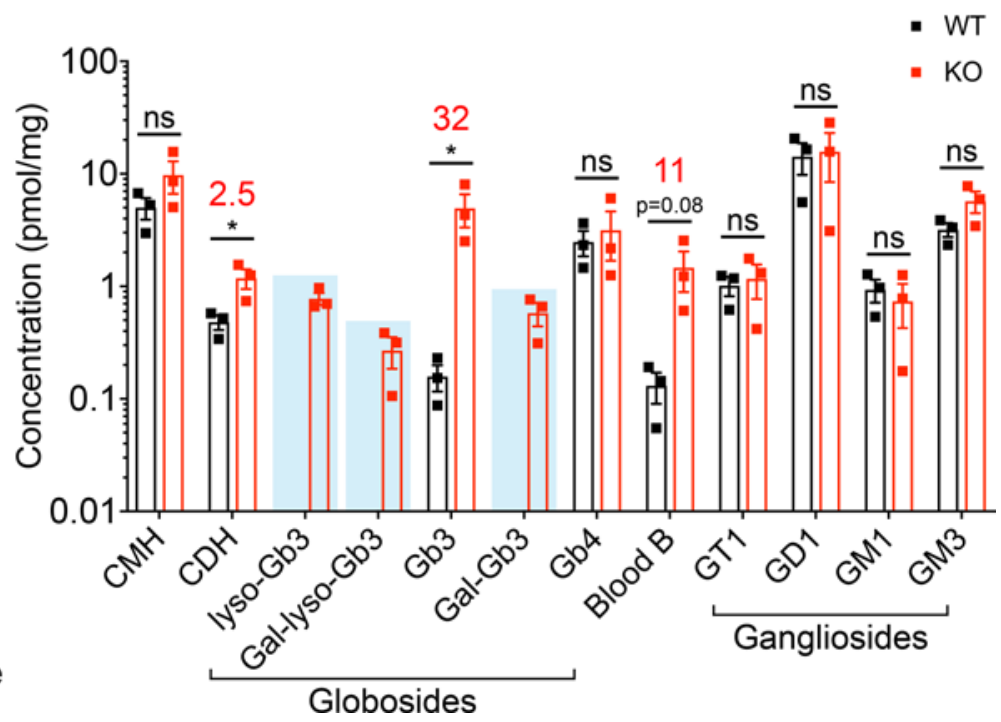

Figure 3. Glycosphingolipids (GSLs) in Fabry rat serum and RBCs. GSLs were extracted from serum (A) and RBC (B) from 13-week-old male rats ( $n=3$ WT, $n=3 \mathrm{KO}$ ). Following purification and permethylation, GSLs were analyzed and quantified by nanospray ionization-mass spectrometry. Note the log scale on the $y$ axis in B. Shown are mean \pm SEM, and means were compared using unpaired, 2-tailed $t$ tests to determine genotype differences in GSLs. If GSL means in KO rats were statistically elevated above WT means, the fold increase is shown above in red. GSLs detected in KO, but not WT, serum and RBCs are highlighted with light blue boxes. Lyso-Gb3, globotriaosylsphingosine; Gb3, globotriaosylceramide; Gb4, globotetraosylceramide; GM3, monosialoganglioside GM3; CMH, ceramide monohexoside; CDH, ceramide dihexoside; Gal, galactose; GT1, trisialoganglioside GT1; GD1, disialoganglioside GD1; GM1, monosialoganglioside GM1. ${ }^{*} P<0.05,{ }^{* *} P<0.001$.

Comparing WT and KO rat serum, there were no significant differences in the levels of globotetraosylceramide (Gb4) or monosialoganglioside GM3, which are GSLs that do not contain terminal $\alpha$-galactose, thus serving as controls (Figure 3A). These data demonstrate that the serum GSL accumulation pattern in the Fabry rat is similar to what is seen in patients.

Next, we looked at whether these biomarkers are also increased in RBC because globo-series GSLs are highly abundant in this cell type. In RBC, ceramide dihexoside (composed of lactosylceramide and galabiosylceramide, which are isotopic GSLs; Figure 1) and Gb3 were significantly elevated in KO compared with WT at 2.5- and 32-fold, respectively (Figure 3B). Although not statistically significant $(P=0.08)$, a trend toward increased blood group B levels was observed in KO RBC compared with WT (Figure 3B). GSLs - including lyso-Gb3, lyso-Gb3 with a galactose extension, and $\mathrm{Gb3}$ with a galactose extension - were detected in KO, but not WT, RBC (Figure 3B, blue boxes). There were no significant differences in GSL species that do not contain terminal $\alpha$-galactose, such as ceramide monohexoside, Gb4, or gangliosides, measured in WT and KO RBC (Figure 3B). In summary, analysis of serum and RBC GSL profiles demonstrate that Gla-KO rats accumulate the established biomarkers, Gb3, lyso-Gb3, along with blood group B, as do patients with Fabry disease.

Fabry rat DRG accumulate Gb3 species. Neuropathic pain is usually the first symptom experienced by patients with Fabry disease. As pain can be centrally or peripherally mediated, we analyzed the GSLs in both Fabry rat brain and DRG. There was no appreciable difference in the brain GSL profile from WT and KO males as evaluated by TLC (Figure 4A). NSI-MS analysis detected no differences in brain Gb3, but lyso-Gb3 was elevated 26-fold in KO compared with WT brain (Figure 4B). No differences were detected in all other brain GSLs that do not contain terminal $\alpha$-galactose, including ceramide monohexoside, sulfatide, and ganglioside species (Figure 4B). TLC analysis of GSLs extracted from lumbar DRG showed an intense doublet that comigrated with the Gb3 standard in the KO male and HET and KO female samples (Figure 4C). Polygalactosylated Gb3 species in HET and KO DRG were also seen as slower migrating signals compared with Gb3 (Figure 4C). By NSI-MS, lyso-Gb3, Gb3, and Gb3 with a galactose extension were all found to be significantly elevated in KO DRG, at 50-, 130-, and 25-fold, respectively (Figure 4D). Digestion with linkage-specific $\alpha$-galactosidase confirmed that the polygalactosylated Gb3 species contained terminal $\alpha$-linked galactose residues (Supplemental Figure 1; supplemental 
A

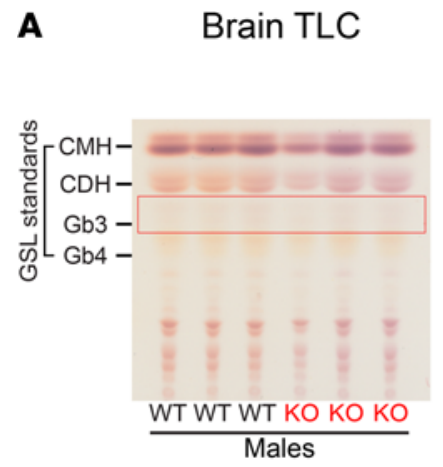

C Dorsal root ganglia TLC

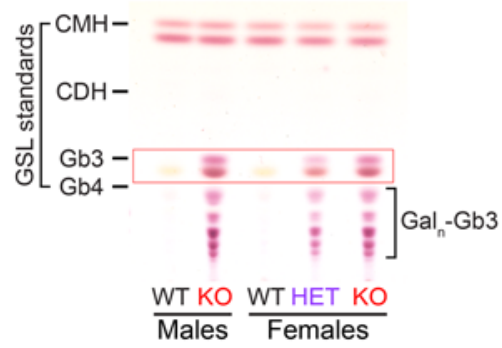

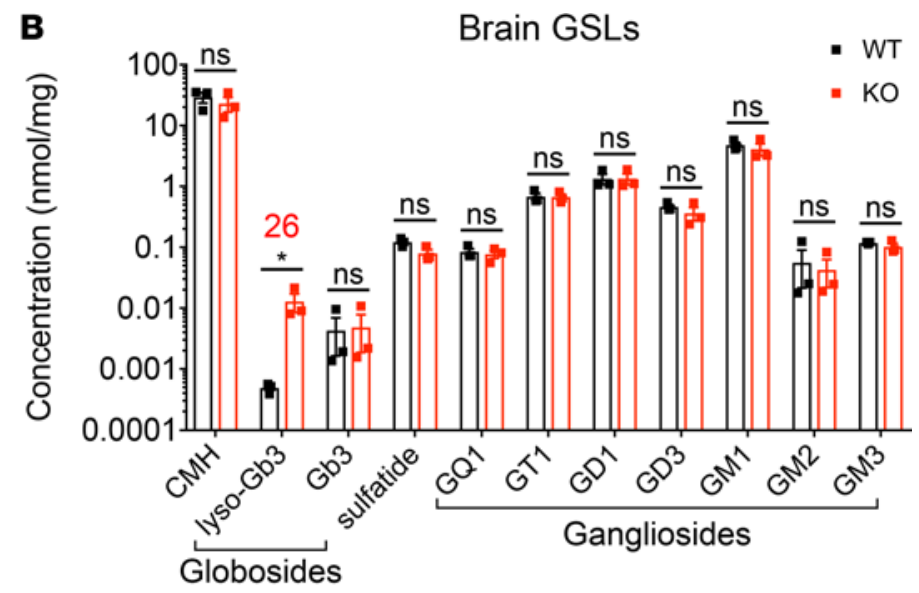

D

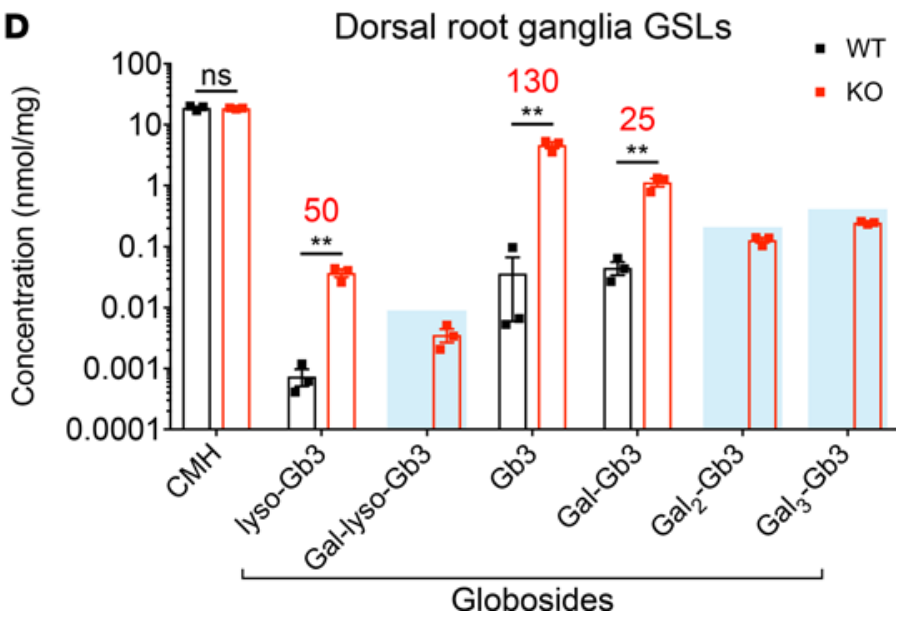

Figure 4. Glycosphingolipid (GSL) storage in Fabry rat brain and dorsal root ganglia. (A) Extracted GSLs from 13-week-old male rat brains ( 3 WT, 3 KO) were analyzed by TLC using a solvent system of chloroform/methanol/water (60:40:10, v/v/v). (B) GSLs from the 3 WT and 3 KO male brains in A were quantified by nanospray ionization-mass spectrometry (NSI-MS) (note the log scale on the $y$ axis). (C) GSLs were extracted from 13-week-old dorsal root ganglia (DRG) and were analyzed by TLC using a solvent system of chloroform/methanol/water (60:35:8, v/v/v). (D) NSI-MS was used to quantify GSLs from WT and KO DRG (note the log scale on the $y$ axis). WT DRG quantification consists of 2 WT males and 1 WT female, and KO DRG quantification consists of $2 \mathrm{KO}$ males and $1 \mathrm{KO}$ female. Gb3 species are outlined with the red box in $\mathbf{A}$ and $\mathbf{C}$. In $\mathbf{B}$ and $\mathbf{D}$, mean \pm SEM are shown and GSL means from WT and $\mathrm{KO}$ samples are compared using unpaired, 2-tailed $t$ tests. If a significant difference in mean is detected, the fold increase in KO GSL is shown above in red. GSL species detected in KO, but not WT, DRG are highlighted with light blue boxes. CMH, ceramide monohexoside; CDH, ceramide dihexoside; Gb3, globotriaosylceramide; Gb4, globotetraosylceramide; lyso-Gb3, globotriaosylsphingosine; GQ1, tetrasialoganglioside GQ1; GT1, trisialoganglioside GT1; GD1, disialoganglioside GD1; GD3, disialoganglioside GD3; GM1, monosialoganglioside GM1; GM2, monosialoganglioside GM2; GM3, monosialoganglioside GM3; Gal, galactose; HET, heterozygous. ${ }^{*} P<0.05,{ }^{* *} P<0.01$.

material available online with this article; https://doi.org/10.1172/jci.insight.99171DS1). The linkage position of these terminal galactose residues was confirmed to be $\alpha 1-3$ by detecting instructive cross-ring cleavage fragments in $\mathrm{MS}$ with multidimensional fragmentation $\left(\mathrm{MS}^{\mathrm{n}}\right)$ spectra (Supplemental Figure 2) (12). In addition, lyso-Gb3 with a galactose extension, Gb3 with 2 galactose extensions, and Gb3 with 3 galactose extensions were detected in KO, but not WT, DRG (Figure 4D, blue boxes). To summarize, KO brain only shows significant elevation of lyso-Gb3, but KO DRG show significantly elevated Gb3, lyso$\mathrm{Gb3}$, and polygalactosylated $\mathrm{Gb3}$ species.

Significant $N$-glycan changes are observed in Fabry rat DRG. To further investigate molecular changes in Fabry DRG, we assessed protein N-glycosylation in KO and WT DRG by NSI-MS ${ }^{\mathrm{n}}$. In KO and WT DRG, we identified unique hybrid glycans carrying both sulfate and sialic acid whose abundances were decreased in the KO compared with WT, as were the abundances of other hybrid and complex N-glycans (Figure 5, Supplemental Figure 3, and Supplemental Table 1). For the high mannose (Man) type glycans whose masses are well resolved from other glycans in full MS analysis (Man6, Man7, Man8, and Man9, highlighted as magenta peaks in Figure 5), little change in abundance was observed between WT and KO DRG (Figure 5). The 


\section{Dorsal root ganglia N-glycans}
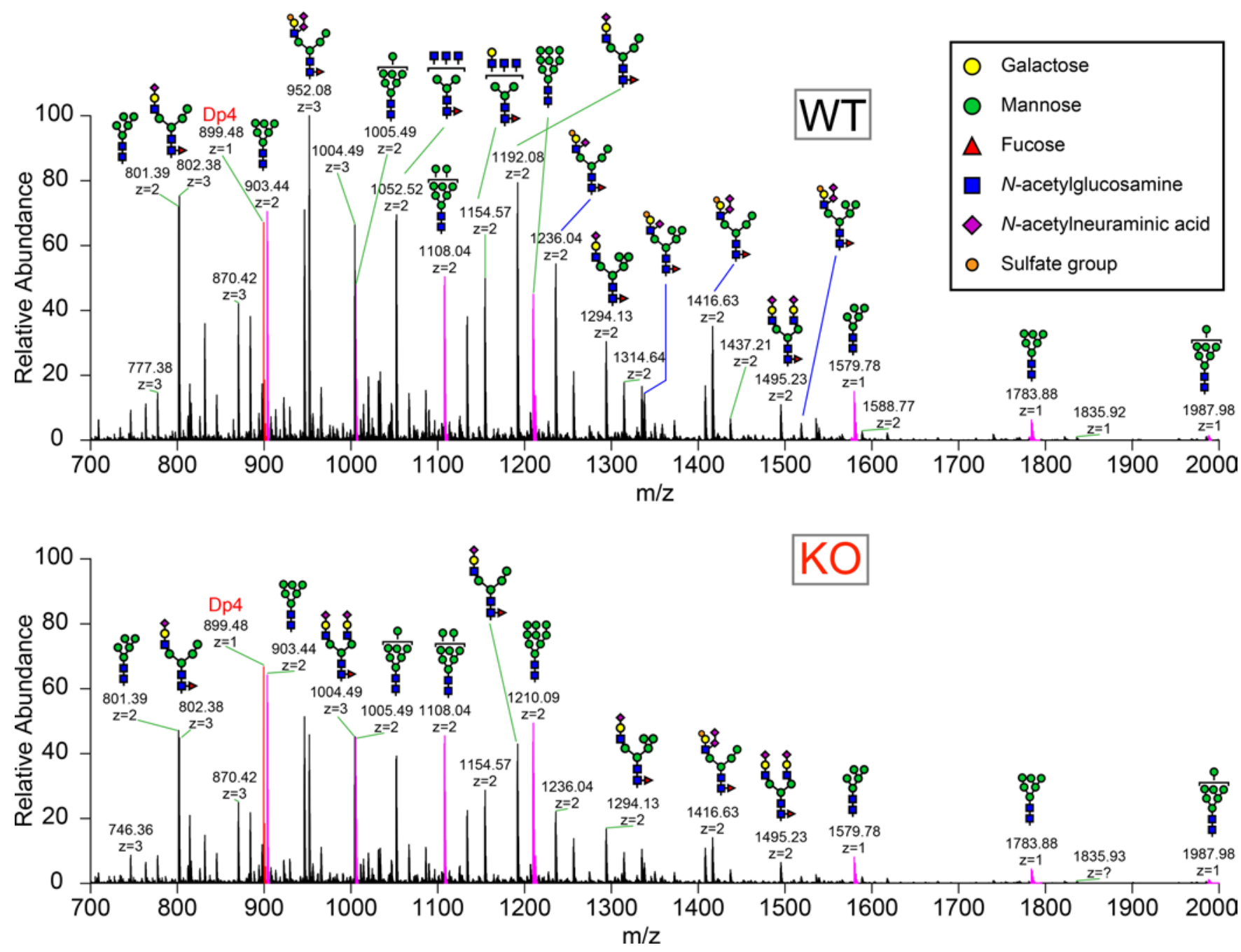

Figure 5. Complex and hybrid $\mathbf{N}$-glycans are decreased in Fabry rat dorsal root ganglia (DRG). N-glycans were prepared from DRG obtained from males at 13 weeks of age. Full mass spectra from WT (top) and KO (bottom) are shown. Peaks colored magenta report the abundance of the annotated high-mannose glycans. Structural assignments for complex and hybrid $\mathrm{N}$-glycans are based on collision-induced dissociation fragmentation performed in nanospray ionization-tandem mass spectrometry analyses of the released, permethylated glycans. The maltotetraose (Dp4) peak corresponds to a standard permethylated glycan that was added to both samples to give the same final concentration, providing a reference for comparing glycan quantities. The sulfated, disialylated hybrid structure, detected at $\mathrm{m} / \mathrm{z} 1416(\mathrm{~m}+\mathrm{Na})^{2+}$ and $952(\mathrm{~m}+\mathrm{Na})^{3+}$ is one of the glycans that exhibits a striking decrease in Fabry rat DRG (Structure 19 in Supplemental Table 1 and Supplemental Figure 3, fragmented in Supplemental Figure 4).

striking decrease in processed N-glycans (hybrid and complex) without a concomitant decrease in high Man glycan abundance indicates that the impact of Fabry disease on protein glycosylation is primarily at the level of Golgi processing, not on core glycosylation in the endoplasmic reticulum. The structures of the unique sulfo, sialylated N-glycans were confirmed by $\mathrm{MS}^{\mathrm{n}}$ analysis (Supplemental Figure 4) (12). Because N-glycans were not found to differ in brain or serum (Supplemental Figures 5 and 6, respectively), N-glycan aberrations may be unique to Fabry rat DRG. The altered DRG N-glycan profile indicates that, in addition to GSL storage, glycoprotein glycosylation changes may also contribute to the pathogenesis of pain in Fabry disease.

Fabry rat sensory neuron cell bodies are swollen with inclusion bodies. The pronounced GSL accumulation in Fabry rat DRG prompted us to next microscopically examine DRG sensory neurons. Lumbar DRG sections were stained with isolectin B4 (IB4), which binds to glycoconjugates containing terminal $\alpha$-galactose $(13,14)$. KO DRG sensory neurons had increased IB4 staining intensity compared with WT (Figure 6, A and B). The staining appeared punctate in WT neurons compared with a more diffuse pattern apparent in KO neurons (Figure 6B). The staining intensity and neuron soma size also increased in aged $\mathrm{KO}$ (90 weeks) 

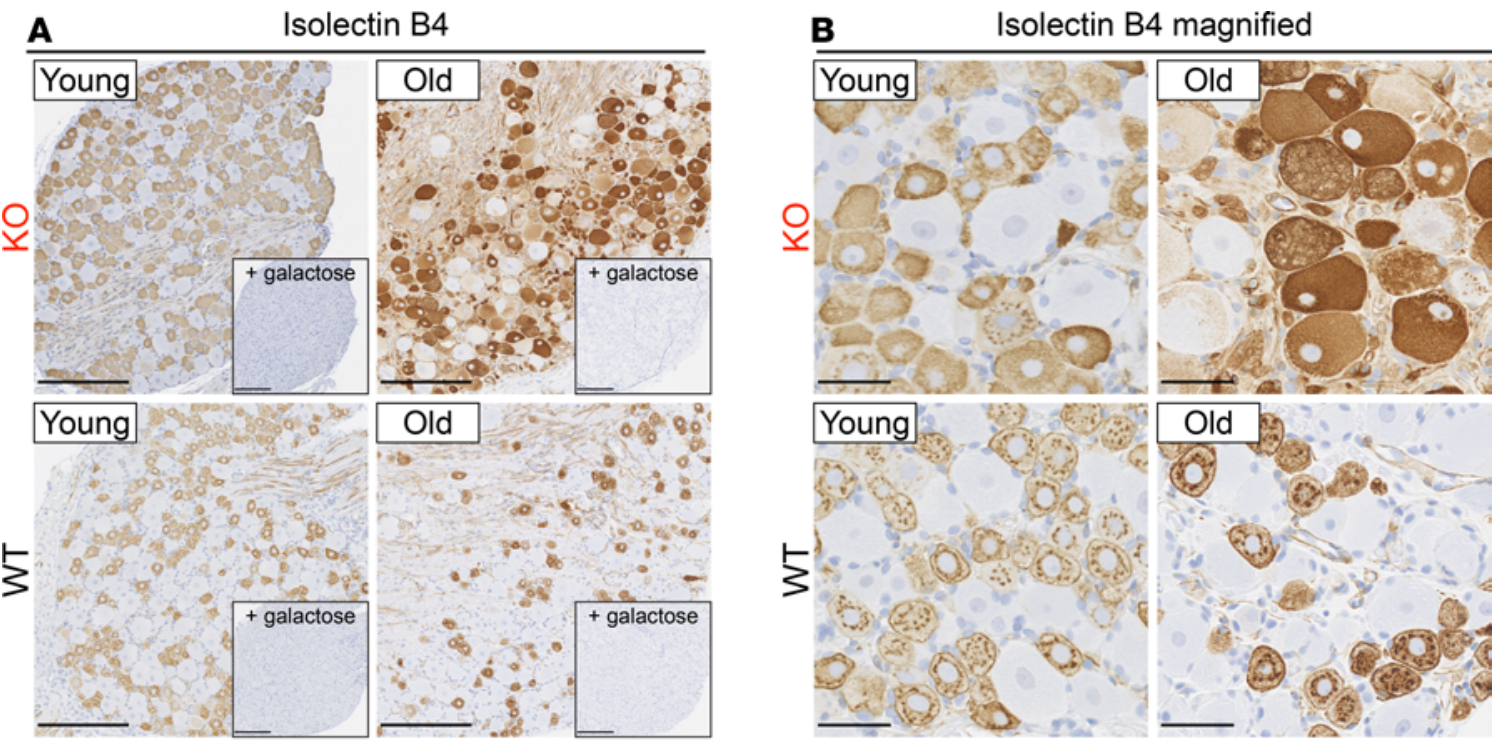

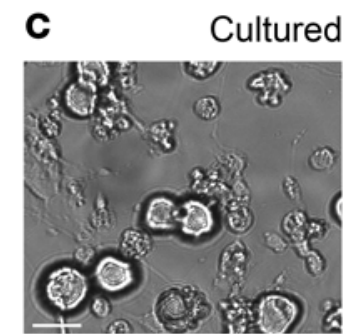

WT
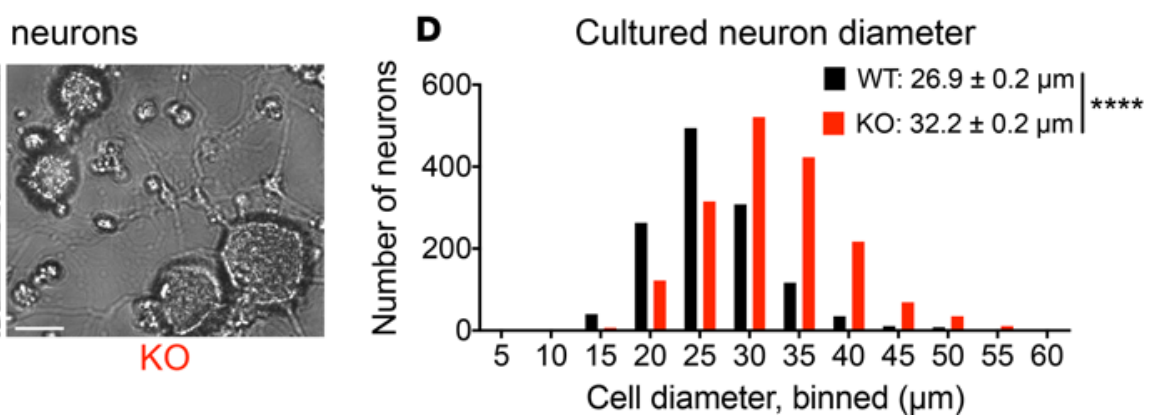

Figure 6. Fabry rat dorsal root ganglia (DRG) sensory neurons are swollen with substrates containing terminal $\alpha$-galactose. (A) Paraffin sections from DRG were stained with Griffonia simplicifolia isolectin B4 at young (13 weeks, 2 WT and 2 KO) and old (90 weeks, 3 WT and 3 KO) ages. Representative images are shown. Insets show isolectin B4 in the presence of $500 \mathrm{mM}$ galactose as a lectin specificity control. (B) Isolectin B4 stained sections as in A, but magnified. (C) Representative brightfield images of cultured DRG neurons. (D) Histograms showing the binned cell diameter frequency of cultured DRC neurons from 3 WT and 3 KO male rats between 19-21 weeks. Inset shows mean cell diameter \pm SEM, and means were compared with an unpaired, 2-tailed $t$ test. Scale bars represent $250 \mu \mathrm{m}(\mathbf{A}), 50 \mu \mathrm{m}$ (A inset and $\mathbf{B})$, and $30 \mu \mathrm{m}$ (C). ${ }^{*{ }^{* *}} \mathrm{P}<0.0001$.

compared with young KO (13 weeks) sensory neurons (Figure 6, A and B). IB4 was also applied to the DRG sections in the presence of $500 \mathrm{mM}$ galactose to demonstrate lectin specificity, and no staining was observed (Figure 6A, insets). Note that IB4 staining was used to demonstrate sensory neuron $\alpha$-galactosyl GSL accumulation rather than to classify neurons based on IB4 staining. Classifying neurons as IB4-positive or -negative is confounded in Fabry disease models, given the accumulation of $\alpha$-galactosyl GSLs, which are ligands for IB4. Nevertheless, the IB4 staining provides striking visual evidence of the neuronal GSL storage in Fabry rats.

In order to quantitatively assess soma size, we cultured DRG neurons from Fabry rats at $\sim 20$ weeks of age. Compared with WT, we found that cultured KO neurons appeared duller and the cytoplasm appeared more granular in brightfield view (Figure 6C). Corroborating the apparent somata enlargement in DRG sections (Figure 6B), we found that cultured KO neurons had significantly larger soma diameters than WT (Figure 6D). Consistent with the DRG TLC and MS analyses (Figure 4, C and D), the IB4 staining and cultured neuron diameter measurements demonstrate that $\mathrm{KO}$ rat sensory neurons accumulate $\alpha$-galactosyl glycoconjugates to a much greater degree than WT neurons, which likely causes swelling of the soma membrane.

Toluidine blue staining of lumbar DRG revealed massive amounts of intensely stained inclusions in KO sensory neuron somata (Figure 7A). The inclusions were more highly prevalent in somata with smaller diameters; many small-diameter somata have C-fiber axons and are nociceptors. In contrast to KO sensory neurons, the cytoplasm of WT sensory neurons appeared homogeneous, and the somata were spared from the presence of inclusion bodies. The DRG inclusions were further evaluated by electron microscopy (Figure 7B). Few electron dense structures were detected in WT sensory neuron somata; however, numerous electron 
A Toluidine blue

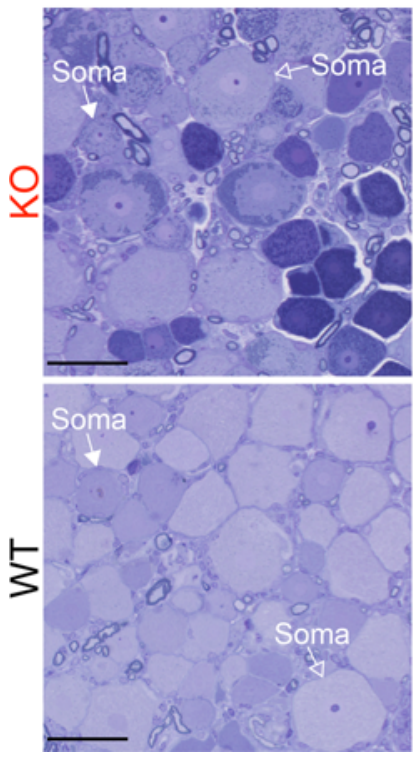

B

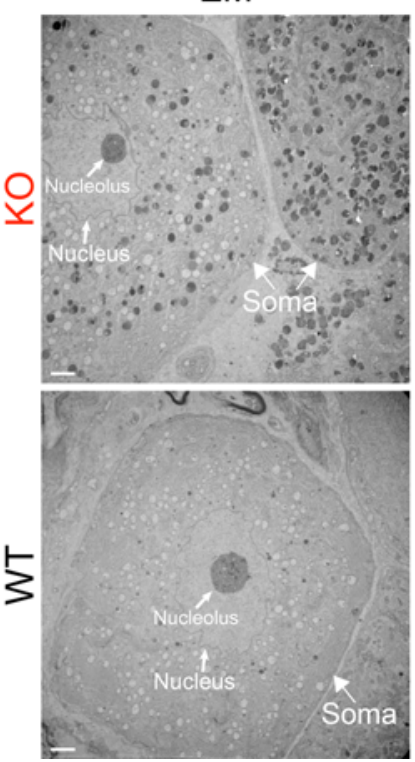

C

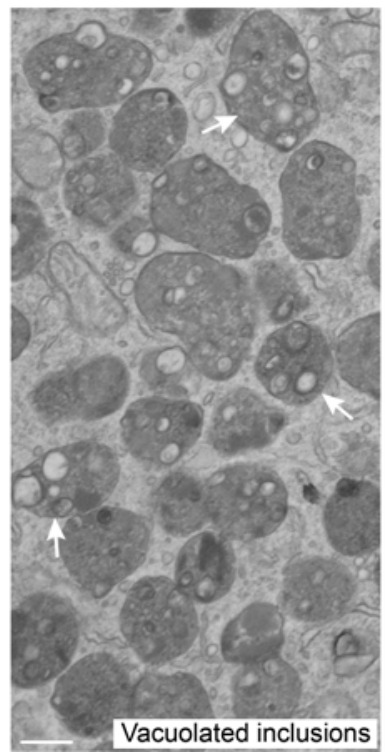

EM magnified - KO

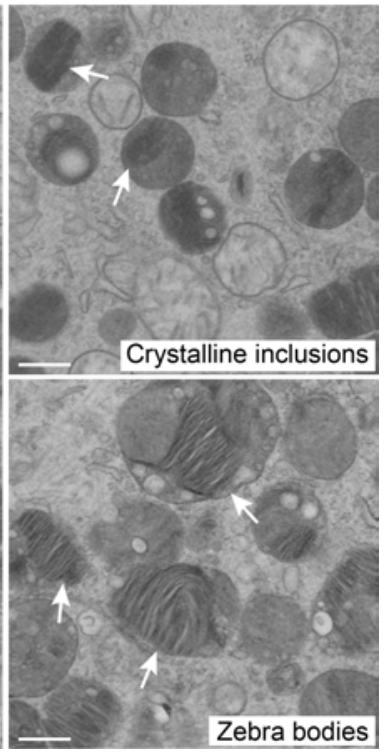

Figure 7. Fabry rat dorsal root ganglia (DRG) sensory neurons are inclusion laden. (A) Semi-thin sections from plastic-embedded DRG from rats at 13 weeks were stained with toluidine blue and representative images (from 2 WT males, 3 KO males, 2 WT females, 2 HET females, and 2 KO females) are shown. Examples of small (closed arrow) and large (open arrow) diameter soma are shown. (B) Representative DRG sensory neuron electron microscopy images from $3 \mathrm{KO}$ and 2 WT males at 13 weeks are shown. (C) Magnified electron microscopy images of KO sensory neurons showing various inclusion morphologies. Scale bars represent $50 \mu \mathrm{m}$ (A), $2 \mu \mathrm{m}$ (B), and $500 \mathrm{~nm}$ (C).

dense inclusions were present in $\mathrm{KO}$ sensory neurons. These inclusions occupied most of the sensory neuron cytoplasm with complete sparing of the nuclei. When the electron dense inclusions in KO sensory neurons were examined at a higher magnification (Figure 7C), various inclusion morphologies were appreciated. Some inclusions were heterogeneous and vacuolated or were filled with material assuming crystalline-like structures. Other inclusions assumed a lamellar morphology (zebra bodies). This finding is characteristic of GSL storage within lysosomes and is classically seen in Fabry patient DRG somata (15). Taken together, these data demonstrate that Fabry rat DRG sensory neurons are inclusion laden, with smaller diameter sensory neurons most prominently affected. These findings are consistent with a small fiber neuropathy, which is experienced by patients with Fabry disease (16).

Fabry rats exhibit altered behavior to mechanical touch and sharp force. Because patients report pain as one of the most debilitating disease symptoms, we next determined if Fabry rats compared with WT rats exhibited response differences to somatosensory stimuli. Male and female rats were longitudinally subjected to 3 established behavioral assays to measure sensitivity to mechanical touch (von Frey up-down test), sharp noxious force (needle test), and cold (dry ice test) (17-21). These tests were performed at 7 different time points from weaning to 1 year of age. Additionally, 1-year-old rats were subjected to the Hargreaves test to assess heat sensitivity (22).

To measure behavioral differences to mechanical touch, von Frey filaments were applied to the plantar surface of the hind paws in order to determine the $50 \%$ withdrawal thresholds. KO males had overall decreased withdrawal thresholds, most prominent at 51 weeks (Figure 8A, top). Significant decreases in withdrawal thresholds were detected in aged $\mathrm{KO}$ females, also with the greatest decrease observed at 51 weeks (Figure 8A, bottom). No significant differences in withdrawal thresholds were detected between WT and HET females. These findings indicate that $\mathrm{KO}$ male and female rats have increased sensitivity to tactile stimuli compared with WT controls, as judged by decreased paw withdrawal thresholds.

Rat hind paws were also probed with a spinal needle to measure differences in response to noxious pin prick. No difference in response (e.g., paw stomping, lifting, or licking) frequency was observed in male or female rats at 4 and 6 weeks (Figure $8 \mathrm{~B}$ ). As the males aged, the $\mathrm{KO}$ rats had increased response frequencies at 10,25, and 51 weeks, reflecting increased response to painful force (Figure 8B, top). Similarly, female KO rats showed increased needle response frequencies at older ages, which were significant at 12, 38, and 51 weeks (Figure 8B, bottom). When the responses are represented by category, aging $\mathrm{KO}$ males and females 

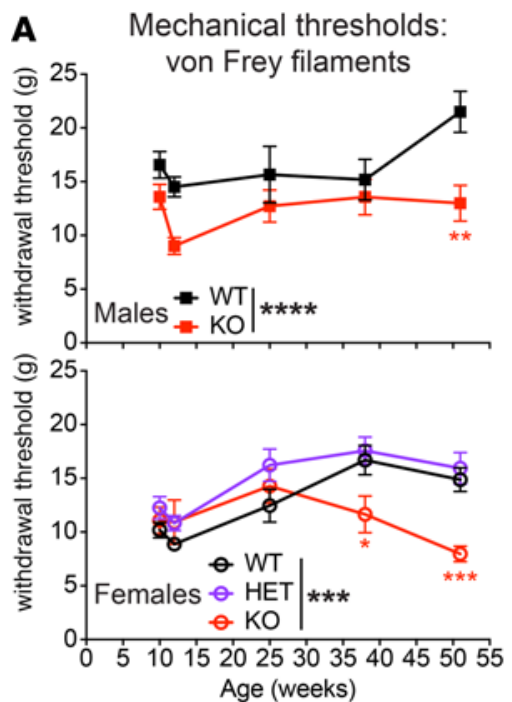

B Sensitivity to noxious force:
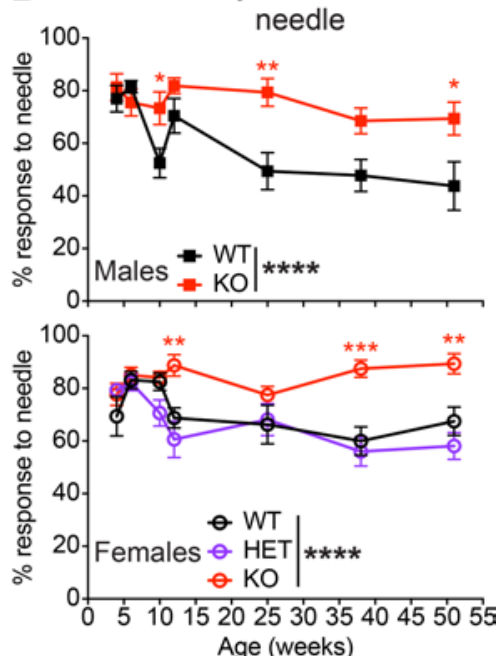

Figure 8. Fabry rats are more sensitive to mechanical touch and noxious force. (A) The von Frey up-down method was performed on WT and KO male and WT, heterozygous (HET), and KO female rat hind paws, and withdrawal thresholds were compared. (B) A 25-gauge spinal anesthesia needle was applied to rat hind paws 10 times, and the percent response to the needle was recorded. (C) At each time point $(4,6,10,12,25,38$, and 51 weeks), the frequency of response type to the needle is shown. A and $\mathbf{B}$ show mean \pm SEM and include $\geq 8$ individual rats in each data point. See Supplemental Tables 3 and 4 for the precise number of animals studied at each time point. Two-way ANOVA was performed on $\mathbf{A}$ and $\mathbf{B}$ to determine the overall genotype effect, and the significance is shown to the right of each legend. To determine genotype differences at each time point studied, multiple comparisons with Bonferroni's correction were performed. ${ }^{*} P<0.05,{ }^{* *} P<0.01,{ }^{* * *} P<0.001$, ${ }^{* * * *} P<0.0001$.
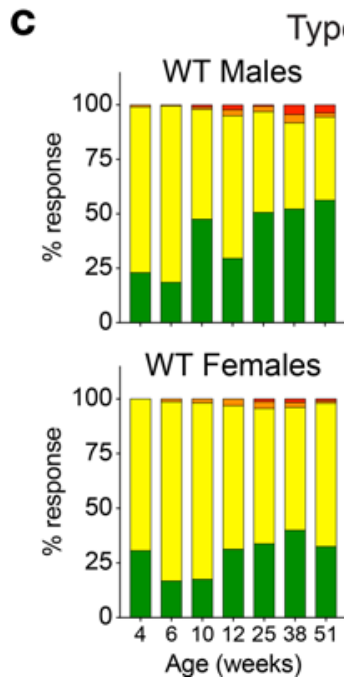
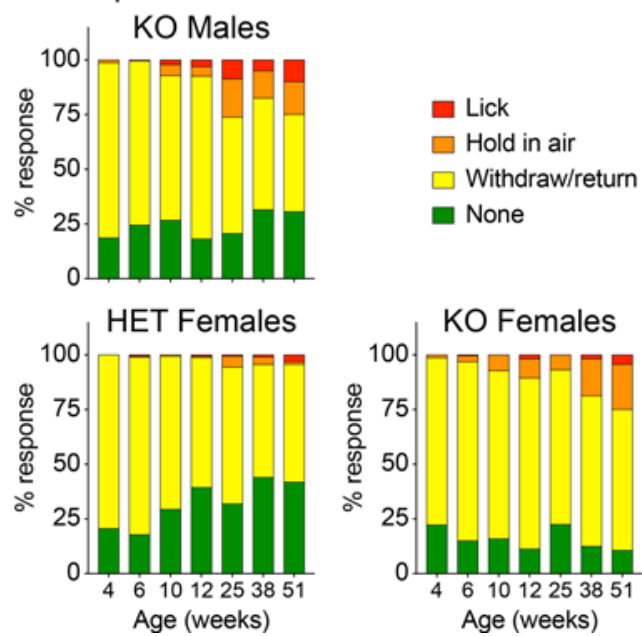

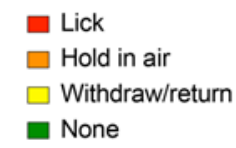

Age (weeks)

had more hyperalgesic responses (e.g., sustained elevation and licking the paw) in the needle test (Figure $8 \mathrm{C}$, orange and red categories, Supplemental Table 2). Thus, both $\mathrm{KO}$ male and female aging rats are more sensitive to a painful mechanical pin prick as compared with WT controls.

Next, we performed thermal assays to assess sensitivity to cold and heat, as patients are known to exhibit cold and warm hyposensitivity $(23,24)$. Compared with WT, neither male nor female Fabry rats showed statistical differences in dry ice reaction latency at any of the ages studied (4-51 weeks) (Supplemental Figure 7A). However, there was a trend toward increased dry ice reaction latency (i.e., cold hyposensitivity) in 51-week-old KO male rats. Aged rats (52 weeks) also showed no behavioral differences to a radiant heat source (Supplemental Figure 7B). Therefore, under our experimental conditions, we did not detect impaired thermal perception in Fabry rats. However, it remains possible that cold and warm hyposensitivity may develop in rats greater than 1 year, as this seems to be the trend in 1-year-old rats (Supplemental Figure 7A). Nevertheless, the presence of mechanical sensitivity with the absence of thermal sensitivity at younger rat ages suggests that mechanical allodynia is an important phenotype to further study, as this may lead to the development of better Fabry pain treatments.

Fabry rat neurons are sensitized to focal mechanical stimulation. The morphological differences in DRG neurons, together with the behavioral data demonstrating that Fabry rats display mechanical sensitization, compelled us to next ask whether KO sensory neurons themselves would be sensitized to direct mechanical probing of the soma membrane. To test this, we utilized whole cell patch clamp recordings together with focal mechanical stimulation of the cell body (Figure 9A). Here, the soma membrane is 
A Mechanical B Stimulation
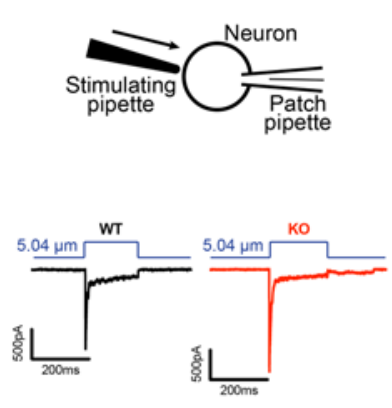

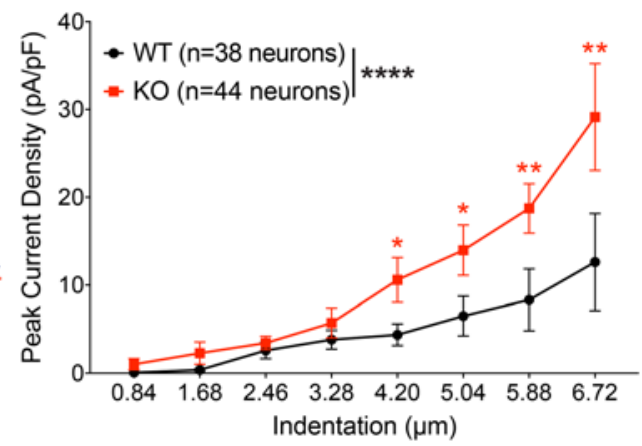

$c$ Mechanical
Threshold

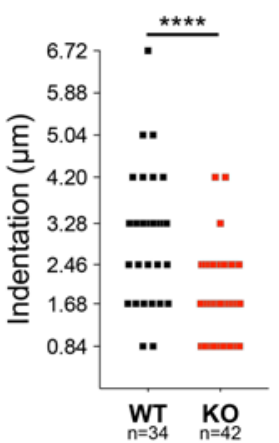

D

Capacitance

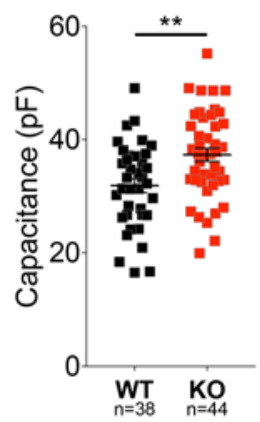

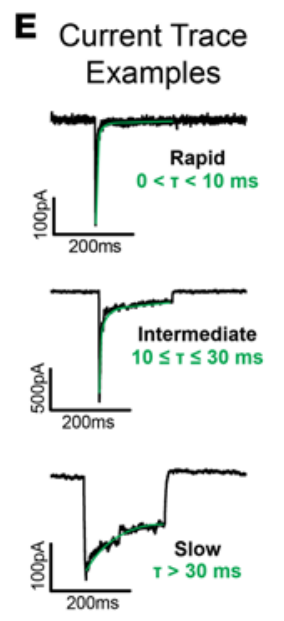

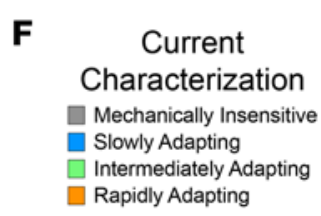

G

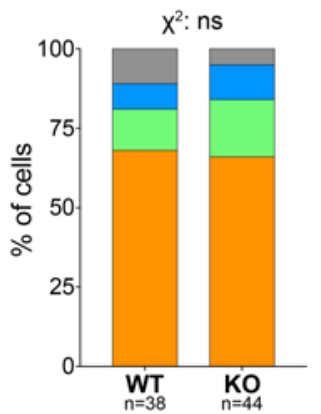

G Neuron

Fragility

$\square$ Auto-patch

a $G \Omega$ seal

$\mathrm{X}^{2: * \star \star}$

Fisher's exact: ***

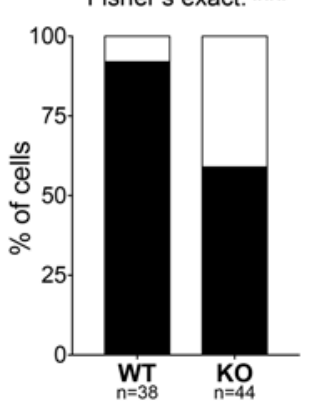

H
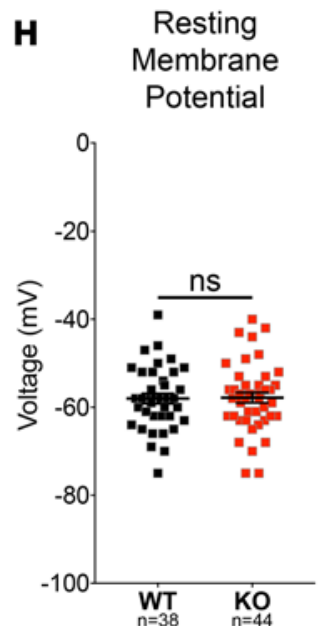

Rheobase

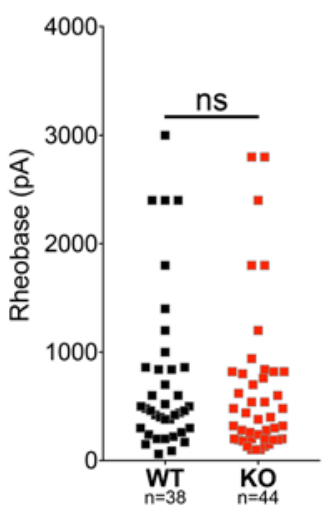

Figure 9. Fabry rat sensory neurons exhibit larger mechanically activated currents than WT. (A) Cultured primary sensory neurons were patched, and a stimulating pipette was used to poke the neuron cell membrane. Below the schematic are example current traces from $1 \mathrm{WT}$ (black) and $1 \mathrm{KO}$ (red) neuron, both indented $5.04 \mu \mathrm{m}$. (B) Patched WT and KO neurons were indented with the stimulating pipette in increasing distances (0.84-6.72 $\mu \mathrm{m})$, and the resulting current density (current normalized to cell capacitance) was recorded. The total respective number of WT and KO neurons patched: 38 and $44(0.84 \mu \mathrm{m}) ; 38$ and $44(1.68 \mu \mathrm{m}) ; 38$ and $42(2.46 \mu \mathrm{m}) ; 35$ and $34(3.28 \mu \mathrm{m}) ; 31$ and $22(4.20 \mu \mathrm{m}) ; 23$ and $17(5.04 \mu \mathrm{m}) ; 13$ and 14 (5.88 $\mu \mathrm{m}) ;$ and 8 and $5(6.72 \mu \mathrm{m})$. Two-way ANOVA with Bonferroni post hoc analysis was performed. (C) The indentation required to elicit the first mechanical current is plotted. WT and KO mechanical threshold medians were compared using a Mann-Whitney test. (D) Neuron capacitance is plotted and means were compared with an unpaired, 2-tailed $t$ test. (E) Example traces showing the different type of inward currents observed in sensory neurons. (F) Current profiles are shown for WT and KO neurons and were compared with a $\chi^{2}$ test. (G) For whole cell patch clamping, the goal is to first form a giga-ohm $(G \Omega)$ seal between the cell and patch pipette before breaking into the cell. Neuron fragility was judged on whether the patch pipette automatically broke into the cell rather than first forming the $G \Omega$ seal. Data were compared with $\chi^{2}$ and Fisher's exact tests. (H) Resting membrane potentials are plotted for both WT and KO neurons, and means were compared with an unpaired, 2-tailed $t$ test. (I) Rheobase, which is the amount of current required to elicit the first action potential, is plotted for both WT and KO and were compared with a Mann-Whitney test. B, D, and $\mathbf{H}$ show mean \pm SEM. Sensory neurons were cultured from 3 WT and $3 \mathrm{KO}$ rats ranging from $19-21$ weeks of age. ${ }^{*} P<0.05,{ }^{* *} P<0.01,{ }^{* * *} P<0.001,{ }^{* * * *} P<0.0001$

used as a model of the peripheral terminal membrane. Given that sensory neuron somata with smaller diameters seemed to be most affected (Figures 6 and 7), we were especially interested in testing these sensory neurons. Thus, we chose to patch neurons that were $32 \mu \mathrm{m}$ or less in diameter, as these generally correspond to somata of $\mathrm{C}$ and A $\delta$ nociceptors (25). Furthermore, we chose to use lumbar DRG from $\sim 20$-week-old male rats, ages at which we observed mechanical hypersensitivity (Figure $8 \mathrm{~A}$ ).

To determine if $\mathrm{KO}$ neurons were sensitized to force, we probed the neurons with 8 indentations, increasing in distance by $\sim 0.84 \mu \mathrm{m}$ with each step. We measured the neuron current and normalized to cell capacitance in order to correct for neuron size differences. The resulting current density (peak current divided by cell capacitance) in response to incremental mechanical probing was significantly larger in KO neurons than in WT neurons (Figure 9B), meaning that KO neurons are more responsive to incremental mechanical stimulation. Furthermore, we found that the KO neurons exhibited their first 
A

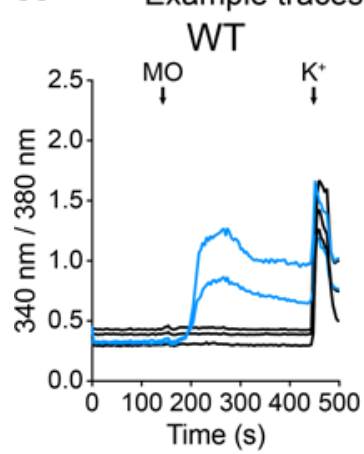

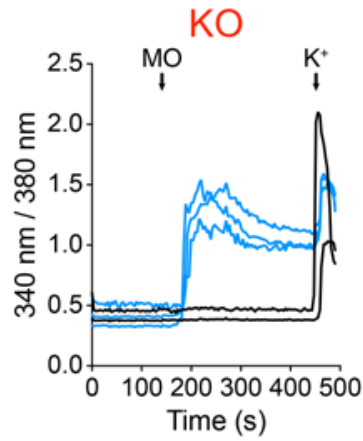

B

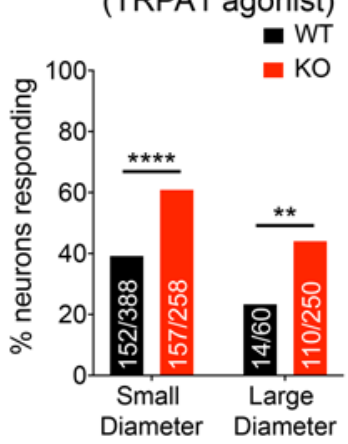

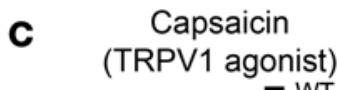

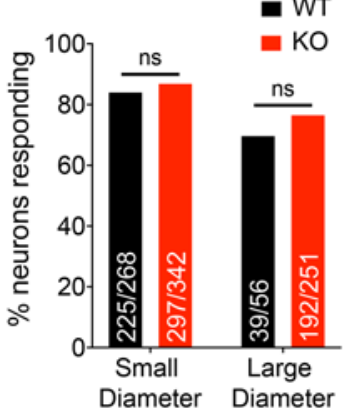

D

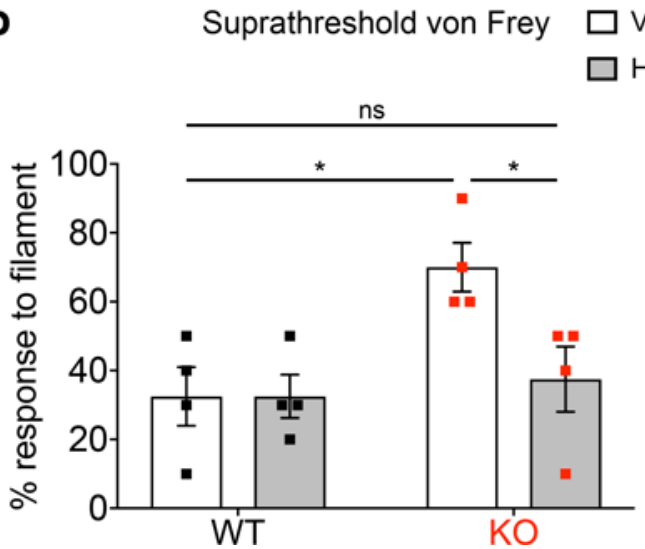

E

Noxious needle $\square$ Vehicle HC-030031

Figure 10. Transient receptor potential ankryin 1 (TRPA1) is a therapeutic target for treating Fabry disease neuropathic pain. (A) Example traces from calcium imaging experiments showing fluorescence ratios $\left(340 / 380 \mathrm{~nm}\right.$ ) with time. Solutions containing allyl isothiocyanate (mustard oil, $\mathrm{MO}$ ) or $\mathrm{K}^{+}$were applied as indicated by the arrows. Traces had a greater than $20 \%$ increase in response to MO are shown in blue. (B) The number of small diameter $(\leq 32 \mu \mathrm{m})$ and large diameter $(>32 \mu \mathrm{m})$ neurons responding to $30 \mu \mathrm{M}$ MO. Numbers of responding neurons and total neurons are shown inside each bar. Neurons are from 3 WT and 3 KO male rats at 19-21 weeks. Fisher's exact test was used to compare WT and KO neuron response percentages. (C) Same as in B, but with $300 \mathrm{nM}$ capsaicin applied. TRPV1, transient receptor potential vanilloid 1. (D) WT and K0 male rats at 35-36 weeks were intraplantarly injected with TRPA1 antagonist, $\mathrm{HC}-030031$, or vehicle ( $n=4$ each group, 16 total rats). An $8.8 \mathrm{~g}$ von Frey filament was applied repeatedly to the hind paw, and the response frequency was determined. Significance was determined using 2-way ANOVA and Bonferroni's post-hoc test. (E) Same as in D, but a needle was applied repeated to the hind paw, and the response frequency was determined. Mean \pm SEM are represented in $\mathbf{D}$ and $\mathbf{E} .{ }^{*} P<0.05,{ }^{* *} P<0.01,{ }^{* * *} P<0.0001$.

mechanically activated currents with significantly smaller indentations than the WT neurons (Figure 9C), suggesting a decreased mechanical threshold. Moreover, the KO neuron capacitance was significantly increased compared with WT, indicating that KO somata can hold greater charge than WT somata (Figure 9D). This is likely because the patched KO cells were larger $(29.7 \pm 0.3 \mu \mathrm{m})$ than WT $(28.4$ $\pm 0.4 \mu \mathrm{m})($ mean \pm SEM), presumably as a result of inclusion-laden somata, a finding consistent with the overall increased size of cultured KO somata vs. WT (Figure 6D). Because capacitance was used to normalize current magnitudes as current density (Figure 9B), the statistical increase in KO capacitance highlights the convincing differences in current density between KO and WT neurons.

To further characterize the functional changes that might occur in KO DRG, we studied the current kinetics in somata. When sensory neurons are mechanically probed, mechanically activated inward currents are elicited, and these can be categorized into 3 subgroups based on their decay kinetics parameters ( $\tau$-value). Figure $9 \mathrm{E}$ shows 3 typical current examples. Mechanically activated currents were categorized as rapidly adapting $(0<\tau<10 \mathrm{~ms})$, intermediately adapting $(10 \leq \tau \leq 30 \mathrm{~ms})$, slowly adapting $(\tau>30 \mathrm{~ms})$, or mechanically insensitive (no current above $20 \mathrm{pA}$ elicited with at least 4 stimulations) $(26,27)$. Analyses of these data showed that proportions of the mechanically activated currents were not significantly different between genotypes (Figure 9F).

Further, we observed that the KO neurons were automatically patched (i.e., patched without the initial giga-ohm $[G \Omega]$ seal formation) at a greater frequency than WT neurons (Figure 9G). Thus, the KO neuron membranes may be more fragile than WT. We also assessed neuronal excitability as a control by measuring 
resting membrane potential and rheobase, the amount of current required to elicit the first action potential. Neither parameter was different between genotypes (Figure 9, H and I). Overall, these data correlate well with the behavior data (Figure 8), which demonstrate that sensory neurons play a role in the mechanical sensitivity observed in Fabry disease and support the argument that the pain observed in Fabry disease, at least in part, is peripherally driven.

TRPA1 mediates the mechanical sensitization in Fabry rats. We sought to determine a molecular basis for the increased mechanical sensitivity seen in Fabry rats (Figure 8) and patch clamp experiments (Figure 9). Because TRPA1 has been shown to be necessary for the full expression of mechanical responses in noninjured tissue (28-34), and because TRPA1 has been shown to be integral to mechanical sensitization in acute and chronic inflammation, nerve injury, and diabetic neuropathy (35-39), we asked whether TRPA1 sensitization contributes to the mechanical hypersensitivity in Fabry disease. Calcium imaging experiments were performed with a moderate dose of allyl isothiocyanate (mustard oil), an established TRPA1 agonist, and neurons were classified as mustard oil responsive or nonresponsive (Figure 10A, blue lines and black lines, respectively). Compared with WT neurons, a statistically larger proportion of small- and large-diameter KO neurons were responsive to mustard oil (Figure 10B), indicating that TRPA1 is sensitized in $\mathrm{KO}$ neurons. We also examined responses to a relative of TRPA1, transient receptor potential vanilloid 1 (TRPV1), a heat receptor that is coexpressed with TRPA1 on many sensory neurons and is involved in mechanical sensitization in some types of injury and disease (40-44). Responses to capsaicin, a TRPV1 agonist, were unaltered (Figure 10C). Together, these data indicate that TRPA1 sensitization is specific and not likely a result of global ion channel sensitization in $\mathrm{KO}$ neurons.

To determine whether TRPA1 mediates the mechanical sensitization in Fabry disease, we performed mechanical and noxious behavioral experiments with intraplantar injection of an established TRPA1 antagonist, HC-030031 $(30,36,45)$. Responses to a repeated supratheshold tactile stimuli were tested 1 hour after antagonist or vehicle injection by applying an $8.8 \mathrm{~g}$ von Frey filament 10 times to the plantar hindpaw and quantifying the frequency of withdrawal responses. Consistent with the results shown in Figure 8 , a significant increase in response frequency to suprathreshold stimuli (Figure 10D) and noxious force (Figure $10 \mathrm{E}$ ) was observed in vehicle-injected $\mathrm{KO}$ rats compared with WT rats. Injection of the TRPA1 antagonist rescued the mechanical hypersensitivity experienced by the $\mathrm{KO}$ animals such that their response frequency was no longer statistically different than vehicle-injected WT animals (Figure 10D). Similar findings were observed in a noxious needle test, where HC-030031 decreased the sensitization in KO rats compared with vehicle $(P=0.06)$ (Figure 10E). Taken together, these data show that antagonism of TRPA1 reversed the sensitized responses seen in KO rats and suggest that TRPA1 may be a therapeutic target in treating the neuropathic pain observed in Fabry disease.

\section{Discussion}

Fabry disease was once thought to be exceptionally rare (46), but recent newborn screening efforts have found incidences ranging from 1:3,000 to 1:10,000 (47-49). These new findings highlight that Fabry disease is significantly underdiagnosed. As Fabry disease is now being added to newborn screening panels in several locations, the number of identified patients is expected to increase greatly. Although $\alpha$-Gal A replacement therapy has been available since the early 2000s $(50,51)$, it is limited by its extreme cost and serious risk of deleterious immune reactions (52-54). Furthermore, enzyme replacement therapy is not effective in some patients, such as those with advanced end-stage organ disease and multiple comorbidities (55), and does not completely alleviate the neuropathic pain. Because pain studies in Fabry mouse models have been conflicting and few in number, our goal was to generate a larger, nonmouse animal model. Here, we show that $\alpha$-Gal A-deficient male and female rats store $\alpha$-galactosyl GSLs in all tissues examined and manifest symptoms similar to those seen in Fabry patients, such as altered touch and pain detection. We discovered that Fabry rat sensory neurons are swollen and inclusion laden, and their mechanical currents are sensitized, implying that primary sensory neurons are key targets to treat Fabry disease pain. Furthermore, we found that the TRPA1 channel is sensitized in Fabry rat sensory neurons, and we show that TRPA1 antagonism is a promising therapeutic avenue for Fabry disease.

Pediatric and adult patients with Fabry disease ( $40 \%$ females, $60 \%-70 \%$ males) experience burning, stabbing, or shooting pain symptoms $(4,56-58)$, suggesting that their pain is neuropathic in nature. Patients mostly experience evoked pain in terms of allodynia (pain to a normally nonpainful stimulus), but many experience chronic pain, crises, and pain attacks that are intensified with exercise, heat, and fever $(4,59)$. 
Neuropathic pain is often described as the most debilitating symptom of Fabry disease and one that begins the earliest in life (60). While the pain is intense in childhood, it may partially subside as patients age, but many adults still suffer from a significant amount of pain (4). In addition to enzyme replacement therapy, Fabry disease pain is currently treated by a variety of medications, including anticonvulsants, tricyclic antidepressants, serotonin-norepinephrine reuptake inhibitors, nonsteroidal anti-inflammatory drugs, and opioids (58). However, many of these drugs have serious side effects, and opioids are notoriously known to lead to dependency and addiction, as seen in the current opioid epidemic. Therefore, animal models that recapitulate patient phenotypes, such as the Fabry rat model, are crucial for the development of better therapies to treat the neuropathic pain experienced by these patients.

Somatosensory phenotypes have been examined in Fabry mice, resulting in a range of observations and interpretations $(8,9,61,62)$. In a recent study, Üçeyler et al. robustly characterized behavioral phenotypes in Fabry mice from 2-27 months and used extensively backcrossed WT controls (10). Consistent with our results, the authors found mechanical hypersensitivity in $\mathrm{KO}$ males and females. However, this study and several other reports required very large numbers of Fabry mice to achieve statistical significance in behavioral experiments. In our longitudinal study of rats from 4-51 weeks, we found that both KO male and female rats develop hypersensitivity to mechanical touch and noxious force with increasing age (Figure 8). Advantages of our somatosensory studies in the Fabry rat include the assessment of all 5 genotypes (including HET females), comparison with littermate WT controls, and the need for relatively few animals per group $(n=4-8)$ to achieve statistical significance. Thus, the rat model will be useful in advancing Fabry disease neuropathic pain research and in clarifying previous mouse studies.

Previous patch clamp studies with DRG neurons from Fabry mice have focused on changes in voltage-gated sodium, potassium, and calcium channels, but not on mechanical sensitivity or mechanotransduction ion channels $(63,64)$. This is despite the fact that mechanical allodynia is a prevalent symptom in patients with Fabry disease (24). To our knowledge, our report is the first study to demonstrate that sensitization of the sensory neuron cell membrane to mechanical probing, as observed by both decreased mechanical thresholds and amplified mechanically activated currents (Figure 9), plays a role in the mechanical hypersensitivity observed in Fabry disease. We also evaluated the function of TRPA1, which has been shown to contribute to mechanical responses in serving as an amplifier of mechanical stimuli under noninjury conditions (29, 31-33). Furthermore, TRPA1 has also been shown to be integral to mechanical sensitization in pain-related disorders stemming from peripheral inflammation, neuropathic pain, and diabetic neuropathy (35-38). Importantly, TRPA1 does not appear to underlie the baseline, protective mechanical nociception in these disorders, but its inhibition alleviates the sensitized aspect of mechanical pain (35). We showed that TRPA1 is sensitized in Fabry rat sensory neurons and that blocking this channel decreases the mechanical and noxious hypersensitivity in Fabry rats (Figure 10). These results demonstrate that TRPA1 is a potential novel candidate target for developing nonopioid therapies for the neuropathic pain of Fabry disease.

The mechanisms driving Fabry disease neuropathic pain are undeniably complex and are only now beginning to be explored. We identified that TRPA1 plays a role in Fabry rat mechanical sensitization, but what causes TRPA1 sensitization in $\alpha$-Gal A-deficient sensory neurons? Three potential contributors to TRPA1 ion channel sensitization and/or neuropathic pain in the context of Fabry disease include: (i) GSL accumulation, (ii) N-glycan alterations, and (iii) physical forces. All of these contributors are non-mutually exclusive.

First, GSL accumulation within sensory neurons likely contributes to Fabry disease pain. Growing evidence supports that lipids modulate the function of ion channels involved in mechanotransduction (65-67), and Sághy et al. recently reported that lipid rafts and sphingomyelin alter the calcium gating properties of TRPA1 channels (68). In the context of Fabry disease, one study found that exogenously applied lyso-Gb3 evoked increased cytoplasmic calcium levels in WT mouse sensory neurons, although the GSL specificity of this response remains to be determined (64). We show that Fabry sensory neurons exhibit dramatic increases in the storage of Gb3 and lyso-Gb3 species (Figure 4), which could impact the function of ion channels within these neurons. In addition to intrinsic storage, it is also reasonable to hypothesize that circulating factors could further sensitize sensory neuron ion channels at the DRG soma level. Because the DRG have large endothelial fenestrations and a rich capillary bed that vascularize the DRG (69), substances in blood, such as Gb3 or lyso-Gb3, may readily have access to the cell bodies within the DRG. Additionally, the DRG in Fabry patients are enlarged and have dysfunctional vascular perfusion $(70,71)$, further supporting that the DRG may be a key pathophysiological site for the development of pain in Fabry disease. 
Second, N-glycan alterations of cell-surface proteins, such as TRPA1, may contribute to neuropathic pain. In Fabry rat DRG, we found decreases in hybrid N-glycans carrying sulfate and sialic acid groups (Figure 5). It is possible that these molecular changes in DRG glycoproteins contribute to the neuropathic pain of Fabry disease. Several studies have shown that N-glycans are critical in regulating TRP channel functions (72-75), including those of TRPA1. Future studies are needed to determine if TRPA1 in Fabry rat sensory neurons has altered N-glycosylation. Our studies, however, identify that — in addition to Gb3 storage driving neuron hypersensitivity - N-glycan changes may also play a role in Fabry disease pain. If true, this knowledge may lead to the development of novel treatments that correct the glycoprotein disturbance, potentially by injecting therapeutics directly into the DRG.

Third, in addition to the biochemical alterations mentioned above, the development of pain may be a result of physical forces within the DRG. It has been shown that DRG are enlarged in patients with Fabry disease (70). Our Fabry rat studies show that DRG sensory somata are also enlarged (Figure 6) and are sensitized to mechanical forces at the soma level (Figure 9). These findings point to another potential explanation of chronic pain in Fabry disease: the swollen sensory neurons place pressure on one another in the confined DRG space and may lead to spontaneous action potential firing. Other studies point to another possible mechanism in which soma swelling alters the tension of the plasma membrane bilayer on embedded mechanotransduction ion channels, resulting in enhanced response to force $(76,77)$. Similarly, membrane fluidity alterations may contribute to Fabry disease pain, as we found that the cell membrane may be more fragile in Fabry rat sensory neurons (Figure 9G). Clearly, more work is needed to elucidate how $\alpha$-Gal A deficiency leads to mechanical pain, but these studies provide suggestions for future investigations of Fabry rat DRG function and physiology.

In summary, we generated a new Fabry disease animal model that mimics the GSL storage and neuropathic pain symptoms observed in patients. We showed that Fabry rat sensory neurons are sensitized to mechanical stimulation of the soma membrane. Finally, we identified that the ion channel, TRPA1, which serves as an amplifier of mechanical stimuli in other pain disorders and diseases, is sensitized in Fabry rat sensory neurons and is a promising drug target for treating Fabry disease pain. We focused our initial Fabry rat studies on somatosensory phenotypes and mechanisms, as neuropathic pain greatly contributes to the overall decreased quality of life in Fabry patients. Studies are underway to characterize other Fabry rat phenotypes seen in patients, including renal, cardiac, and cerebrovascular diseases. We anticipate that this rat model will be highly valuable in defining the complex pathophysiology of Fabry disease and will be helpful in evaluating novel therapeutics in treating disease symptoms such as chronic pain.

\section{Methods}

Animals. Female and male DA rats were acquired from Taconic Biosciences Inc. (DA/MolTac) and were used to generate $\alpha$-Gal A-deficient rats (see below). Rats were maintained on a 12-hour light/dark cycle and were provided with standard laboratory chow (Purina, diet 5001) and drinking water ad libitum.

Generation of Gla-KO rat model. DA-Gla ${ }^{\text {em2Mcwi }}$ (Rat Genome Database ID, 10054398; http://rgd.mcw. $\mathrm{edu} / \mathrm{rgdweb} / \mathrm{report} / \mathrm{strain} / \mathrm{main} . \mathrm{html}$ ?id=10054398) rats were generated using CRISPR/Cas9 technology by disrupting the rat Gla gene (GenBank accession number NM_001108820.3). The CRISPR gRNA target site with PAM sequence (5' - GCCCTTTGAATCCCTCTCGG - 3') was cloned into the pX330-U6-Chimeric_BB-CBh-hSpCas9 dual expression plasmid, which was provided by Feng Zhang (the Broad Institute of MIT and Harvard University, Cambridge, Massachusetts, USA) (Addgene plasmid 42230) (78). Embryo injections were performed as previously described (79). Briefly, DA rat embryos were generated and collected by superovulation of DA females and mating with DA stud males. The CRISPR plasmid was diluted to $5 \mathrm{ng} / \mu \mathrm{l}$ in buffer $(10 \mathrm{mM}$ Tris- $\mathrm{HCl}, 0.1 \mathrm{mM}$ EDTA, $\mathrm{pH} 7.5$, sterile filtered; MilliporeSigma) and was injected into newly fertilized embryo pronuclei. On the same day as the microinjections, healthy-appearing embryos were transferred into pseudopregnant Sprague Dawley female rats from Charles River Laboratories (SD/CRL).

Ear biopsy was collected from founder $\left(\mathrm{F}_{0}\right)$ generation pups at 10-16 days after parturition, and DNA was extracted for PCR amplification of the target site using primers: Forward 5'-CCTAGAGGATTCAGTGTTTTCAGTAAG-3' and reverse 5'-TCAGTGAGCAACTGTTCATGCA-3'. A CEL I-based mutation detection method was used to screen for positive founders (79). Briefly, PCR products were cloned using the TOPO-TA cloning kit (Invitrogen), and individual plasmid clones were sequenced to determine the mutation. A founder male was identified that had a 47-bp frameshift deletion in exon 2 (Rat Genome 
Sequencing Consortium Rnor 5.0 (https://www.hgsc.bcm.edu/other-mammals/rat-genome-project), chrX: 105,301,986-105,302,032: 5' - CCTCTCGGGAGCCATCCAGCAGTCATCTATGCAGAGGTATTCATAAC - 3'). To eliminate any potential off-target events, the founder male was backcrossed to the parental strain. The resulting HET females were crossed with WT DA males to produce WT $\left(G l a^{+/ 0}\right)$ male, KO $\left(\mathrm{Gla}^{-/ 0}\right)$ male, WT $\left(\mathrm{Gla}^{+/+}\right)$female, and HET $\left(\mathrm{Gla}^{+/-}\right)$female experimental rats. HET females and KO males were crossed to produce homozygous $\left(\mathrm{KO}, \mathrm{Gla}^{--}\right)$females. Using the method developed for simple sequence length polymorphism genotyping (80), all experimental animals were genotyped using fluorescently labeled M13 tagged primers.

Enzyme activity assays. Lysosomal enzyme activities were assayed as previously described (81-83). Minor modifications are described in the Supplemental Methods.

Nomenclature. Graphical representations of monosaccharide residues are as shown in the boxed legends for Figure 1, Figure 5, and Supplemental Figure 3 and are consistent with the Symbol Nomenclature for Glycans (SNFG), which has been broadly adopted by the glycomics community (84).

Isolation of GSLs and TLC. The GSL isolation and TLC procedures were as previously described (8587). Modifications are described in the Supplemental Methods.

MS of GSLs and glycoprotein glycans. The preparation of GSLs and glycoprotein glycans for quantitative MS was performed as described previously, and the relevant method details are presented in the Supplemental Methods $(87,88)$. Intact GSLs and released glycoprotein glycans were permethylated with ${ }^{12} \mathrm{C}$-methyliodide prior to MS analysis according to the method of Anumula and Taylor (89). Known amounts of a maltotetraose oligosaccharide (Dp4) were permethylated with ${ }^{13} \mathrm{C}$-methyliodide for use as reference standards for GSL and glycoprotein glycan quantification (88). Permethylated GSLs were analyzed from multiple biological replicates for serum, RBC, DRG, and brain tissue $(n=3$ for WT and $n=3$ for KO). For all samples, GSLs were characterized by MS and by TLC. N-glycans were released from rat glycoproteins by PNGase F digestion and permethylated and quantified as described previously and as detailed in the Supplemental Methods (90). Permethylated N-glycans were analyzed by NSI-MS in positive ion mode and quantified relative to a known amount of external standard (Dp4, permethylated with heavy methyliodide) supplemented into the sample prior to injection. For the characterization of sulfated N-glycans, total ion mapping profiles were filtered with neutral loss of sulfate group (91). N-glycan structures carrying the sulfate group were further subjected to manual $\mathrm{MS}^{\mathrm{n}}$ analysis for complete structural determination. The MS-based glycomics data generated in these analyses and the associated annotations are presented in accordance with the MIRAGE standards and the Athens Guidelines, which include explicit descriptions of instrument settings, fragmentation strategies, and quantification parameters $(92,93)$.

Optimization of lyso-GSL analysis. The free amino group in the sphingoid base of lyso-GSLs significantly suppresses ionization in the mass spectrometer, thereby decreasing detection sensitivity. Permethylation of molecules with a free amino group results in the formation of quaternary ammonium salts that suppress ionization. To circumvent this problem, we developed an improved approach for the structural assignment of lyso-GSL species using a 1-step chemical reaction that results in $\mathrm{N}$-acetylation of the sphingoid base (see Supplemental Methods).

Histology, IHC, and transmission electron microscopy. Tissues were immediately removed from euthanized rats and fixed in 10\% (v/v) neutral buffered formalin (Thermo Fisher Scientific) for 24-72 hours. After fixation, tissues were dehydrated through graded ethanol (Decon Laboratories), cleared with xylene (Leica Biosystems), and paraffin (Sakura) infiltrated and embedded. Tissue blocks were cut into 4- $\mu$ m sections and mounted on poly-L-lysine-coated slides (Thermo Fisher Scientific). Slides were antigen retrieved and stained using standard labeled streptavidin-biotin detection. Biotinylated Griffonia simplicifolia IB4 (Vector Laboratories, B-1205, $5 \mu \mathrm{g} / \mathrm{ml}$ ) followed by incubation with streptavidin-HRP and 3,3'-diaminobenzidine (Dako) was used to detect $\alpha$-galactosyl glycoconjugates. Lectin specificity was demonstrated by applying IB4 in the presence of $500 \mathrm{mM}$ galactose (MilliporeSigma) prior to staining protocol. High-resolution images were obtained with a Hamamatsu NanoZoomer 2.0-HT digital slide scanner.

Electron microscopy studies were conducted on lumbar DRG (L1) that were rapidly removed and placed into fixative $(0.1 \mathrm{M}$ sodium cacodylate buffer containing $2 \%[\mathrm{v} / \mathrm{v}]$ paraformaldehyde and $2 \%[\mathrm{v} / \mathrm{v}]$ glutaraldehyde; Electron Microscopy Sciences) for at least 5 hours at $4^{\circ} \mathrm{C}$. Following fixation, samples were washed 3 times with cacodylate buffer and postfixed with potassium ferricyanide reduced $1 \%(\mathrm{v} / \mathrm{v})$ osmium tetroxide. Tissues were then dehydrated through graded methanol, embedded in EMbed-812 resin 
(Electron Microscopy Sciences), and sectioned at $60 \mathrm{~nm}$. Images were acquired with a Hitachi H600 TEM equipped with a Hamamatsu side entry camera. Additionally, plastic embedded DRG $0.5 \mu \mathrm{m}$ sections were mounted on glass slides and stained with $1 \%(\mathrm{v} / \mathrm{v})$ toluidine blue.

Behavior assays. To assess somatosensory phenotypes in $\alpha$-Gal A-deficient rats, established behavioral assays were performed (17-22). These assays included mechanical thresholds with von Frey filaments (up-down and repeated techniques), sensitivity to noxious force with a repeated needle, cold sensitivity with the dry ice test, and heat sensitivity with the Hargreaves test. A single experimenter conducted all of the behavior assays in Figure 8, while a different experimenter conducted the assays in Figure 10, D and E. Minor modifications to the protocols are described in the Supplemental Methods.

Patch-clamp electrophysiology. Male rats from 19-21 weeks of age were euthanized by isoflurane and thoracotomy. DRG sensory neurons were isolated as previously described (94). Bilateral lumbar DRG (L1-6) were selected because neurons in these ganglia innervate the hind paws. After overnight culture, DRG neurons were used for calcium imaging (below) or patch clamping as previously published (95). Detailed experimental procedures are described in the Supplemental Methods.

Calcium imaging. Coverslips containing DRG sensory neurons (above) were superfused with an extracellular normal HEPES (ENH) buffer, which consists of $150 \mathrm{mM} \mathrm{NaCl}, 10 \mathrm{mM}$ HEPES, $8 \mathrm{mM}$ glucose, 5.6 $\mathrm{mM} \mathrm{KCl}, 2 \mathrm{mM} \mathrm{CaCl}_{2}$, and $1 \mathrm{mM} \mathrm{MgCl}_{2}$ (all compounds from MilliporeSigma). Isolated cells were incubated in the dual wavelength calcium indicator FURA-2 AM, as previously described in Zappia et al. (34). Allyl isothiocyanate (30 $\mu \mathrm{M}$, as referred to as mustard oil) (MilliporeSigma), a TRPA1 agonist, or $300 \mathrm{nM}$ capsaicin (Fluka), a TRPV1 agonist, were used to assess functionality of these channels in WT and KO neurons. A 340/380 ratio that increased by $>20 \%$ from baseline was considered a response to a given stimulus.

Statistics. All statistical analyses were performed with Graph Pad Prism 7 software. An unpaired, 2-tailed $t$ test was used to compare GSL concentrations, cell capacitances, resting membrane potentials, cultured cell diameters, male enzyme activities, and male Hargreaves latencies. One-way ANOVA followed by Dunnett's multiple comparisons was used to compare enzyme activities and Hargreaves latencies for females. Two-way ANOVA was used to determine overall genotype effect of behavior (von Frey, needle, cold tests) and neuron current density, and Bonferroni's multiple comparisons was used post hoc to determine genotype differences at each age or indentation distance, respectively. Similarly, 2-way ANOVA with Bonferroni's multiple comparisons was used to evaluate the effect of HC-030031 on behavioral responses in WT and KO rats. A Mann-Whitney $U$ test was used to compare rheobase values and mechanical thresholds (patch-clamp experiment). A $\chi^{2}$ test was used to analyze needle behavior responses, neuron current characterization, and neuron fragility. Fisher's exact test was also used to assess neuron fragility and neuron response frequencies to mustard oil and capsaicin. Differences were considered significant if $P<0.05$. Asterisks throughout indicate ${ }^{*} P<0.05,{ }^{* *} P<0.01,{ }^{* * *} P<0.001$, and ${ }^{* * * *} P<0.0001$.

Study approval. The IACUC at the Medical College of Wisconsin (Milwaukee, Wisconsin, USA) reviewed and approved all animal protocols. All animal research was carried out in accordance with the recommendations in the Guide for the Care and Use of Laboratory Animals (National Academies Press, 2011).

\section{Author contributions}

JJM, KA, FM, MT, CLS, and NMD designed the research. JJM, KA, FM, CAM, and CLO collected data. JJM, KA, FM, CAM, CLO, MT, CLS, and NMD analyzed and interpreted the data. JJM, KA, FM, MT, CLS, and NMD wrote the manuscript.

\section{Acknowledgments}

We thank Katelyn Sadler for helpful feedback on the manuscript and Anthony Menzel and Angela Beltrame for necropsy, behavior, and neuron culture assistance. We also thank several Medical College of Wisconsin core programs for their help in data acquisition; the Children's Research Institute Histology Core (Christine Duris, Stephanie Wirsbinski, Tatunya Bufford, and Qiuhui Yang) and the Electron Microscopy Core (Clive Wells) provided excellent technical assistance in slide preparation, staining, and imaging, and the Neuroscience Research Center (Research and Education Initiative Fund, Advancing a Healthier Wisconsin) provided facilities to perform behavioral studies. Funding was provided by NIH grants R21NS095627 (to NMD and CLS), R01DK042667 (to NMD), R01NS040538 (to CLS), R01NS070711 (to CLS), R21AI129873 (to KA), P41GM103490 (to MT), and F30DK113641 (to JJM, a predoctoral trainee). JJM is a member of the Medical Scientist Training Program, which is partially supported by T32GM080202. The Gla-KO rat was 
generated under NIH resource grant R24HL114474. The content is solely the responsibility of the authors and does not necessarily represent the official views of the NIH.

Address correspondence to: Nancy M. Dahms or Cheryl L. Stucky, 8701 W. Watertown Plank Road, Milwaukee, Wisconsin 53226, USA. Phone: 414.955.4698, Email: ndahms@mcw.edu (NMD). Phone: 414.955.8373; Email: cstucky@mcw.edu (CLS). Or to: Michael Tiemeyer, 315 Riverbend Road, Complex Carbohydrate Research Center, University of Georgia, Athens, Georgia 30602, USA. Phone: 706.542.2740; Email: mtiemeyer@ccrc.uga.edu.

1. Germain DP. Fabry disease. Orphanet J Rare Dis. 2010;5:30.

2. Desnick RJ, et al. Fabry disease, an under-recognized multisystemic disorder: expert recommendations for diagnosis, management, and enzyme replacement therapy. Ann Intern Med. 2003;138(4):338-346.

3. Vedder AC, et al. The Dutch Fabry cohort: diversity of clinical manifestations and Gb3 levels. J Inherit Metab Dis. 2007;30(1):68-78

4. Üçeyler N, Ganendiran S, Kramer D, Sommer C. Characterization of pain in fabry disease. Clin J Pain. 2014;30(10):915-920.

5. Ohshima T, et al. alpha-Galactosidase A deficient mice: a model of Fabry disease. Proc Natl Acad Sci USA. 1997;94(6):2540-2544.

6. Ohshima T, et al. Aging accentuates and bone marrow transplantation ameliorates metabolic defects in Fabry disease mice. Proc Natl Acad Sci USA. 1999;96(11):6423-6427.

7. Ioannou YA, Zeidner KM, Gordon RE, Desnick RJ. Fabry disease: preclinical studies demonstrate the effectiveness of alpha-galactosidase A replacement in enzyme-deficient mice. Am J Hum Genet. 2001;68(1):14-25.

8. Rodrigues LG, et al. Neurophysiological, behavioral and morphological abnormalities in the Fabry knockout mice. Neurobiol Dis. 2009;33(1):48-56.

9. Lakomá J, Rimondini R, Donadio V, Liguori R, Caprini M. Pain related channels are differentially expressed in neuronal and non-neuronal cells of glabrous skin of fabry knockout male mice. PLoS ONE. 2014;9(10):e108641.

10. Üçeyler N, Biko L, Hose D, Hofmann L, Sommer C. Comprehensive and differential long-term characterization of the alpha-galactosidase A deficient mouse model of Fabry disease focusing on the sensory system and pain development. Mol Pain. 2016;12:1744806916646379.

11. Garman SC, Garboczi DN. The molecular defect leading to Fabry disease: structure of human alpha-galactosidase. J Mol Biol. 2004;337(2):319-335.

12. Ashline D, Singh S, Hanneman A, Reinhold V. Congruent strategies for carbohydrate sequencing. 1. Mining structural details by MSn. Anal Chem. 2005;77(19):6250-6262.

13. Murphy LA, Goldstein IJ. Five alpha-D-galactopyranosyl-binding isolectins from Bandeiraea simplicifolia seeds. J Biol Chem. 1977;252(13):4739-4742.

14. Kirkeby S, Moe D. Binding of Griffonia simplicifolia 1 isolectin B4 (GS1 B4) to alpha-galactose antigens. Immunol Cell Biol. 2001;79(2):121-127.

15. Onishi A, Dyck PJ. Loss of small peripheral sensory neurons in Fabry disease. Histologic and morphometric evaluation of cutaneous nerves, spinal ganglia, and posterior columns. Arch Neurol. 1974;31(2):120-127.

16. Biegstraaten M, Hollak CE, Bakkers M, Faber CG, Aerts JM, van Schaik IN. Small fiber neuropathy in Fabry disease. Mol Genet Metab. 2012;106(2):135-141.

17. Chaplan SR, Bach FW, Pogrel JW, Chung JM, Yaksh TL. Quantitative assessment of tactile allodynia in the rat paw. J Neurosci Methods. 1994;53(1):55-63.

18. Hogan Q, Sapunar D, Modric-Jednacak K, McCallum JB. Detection of neuropathic pain in a rat model of peripheral nerve injury. Anesthesiology. 2004;101(2):476-487.

19. Brenner DS, Golden JP, Gereau RW. A novel behavioral assay for measuring cold sensation in mice. PLoS ONE. 2012;7(6):e39765.

20. Garrison SR, Weyer AD, Barabas ME, Beutler BA, Stucky CL. A gain-of-function voltage-gated sodium channel 1.8 mutation drives intense hyperexcitability of A- and C-fiber neurons. Pain. 2014;155(5):896-905.

21. Weyer AD, Zappia KJ, Garrison SR, O'Hara CL, Dodge AK, Stucky CL. Nociceptor Sensitization Depends on Age and Pain Chronicity(1,2,3). eNeuro. 2016;3(1):ENEURO.0115-15.2015.

22. Hargreaves K, Dubner R, Brown F, Flores C, Joris J. A new and sensitive method for measuring thermal nociception in cutaneous hyperalgesia. Pain. 1988;32(1):77-88.

23. Üçeyler N, et al. Impaired small fiber conduction in patients with Fabry disease: a neurophysiological case-control study. $B M C$ Neurol. 2013;13:47.

24. Torvin Møller A, et al. Functional and structural nerve fiber findings in heterozygote patients with Fabry disease. Pain. 2009;145(1-2):237-245.

25. Oyelese AA, Eng DL, Richerson GB, Kocsis JD. Enhancement of GABAA receptor-mediated conductances induced by nerve injury in a subclass of sensory neurons. J Neurophysiol. 1995;74(2):673-683.

26. Coste B, et al. Piezo1 and Piezo2 are essential components of distinct mechanically activated cation channels. Science. 2010;330(6000):55-60.

27. Hao J, Delmas P. Multiple desensitization mechanisms of mechanotransducer channels shape firing of mechanosensory neurons. J Neurosci. 2010;30(40):13384-13395.

28. Kwan KY, et al. TRPA1 contributes to cold, mechanical, and chemical nociception but is not essential for hair-cell transduction. Neuron. 2006;50(2):277-289. 
29. Kwan KY, Glazer JM, Corey DP, Rice FL, Stucky CL. TRPA1 modulates mechanotransduction in cutaneous sensory neurons. J Neurosci. 2009;29(15):4808-4819.

30. Kerstein PC, del Camino D, Moran MM, Stucky CL. Pharmacological blockade of TRPA1 inhibits mechanical firing in nociceptors. Mol Pain. 2009;5:19.

31. Brierley SM, et al. TRPA1 contributes to specific mechanically activated currents and sensory neuron mechanical hypersensitivity. J Physiol (Lond). 2011;589(Pt 14):3575-3593.

32. Brierley SM, et al. The ion channel TRPA1 is required for normal mechanosensation and is modulated by algesic stimuli. Gastroenterology. 2009;137(6):2084-2095.e3.

33. Vilceanu D, Stucky CL. TRPA1 mediates mechanical currents in the plasma membrane of mouse sensory neurons. PLoS ONE. 2010;5(8):e12177.

34. Zappia KJ, O'Hara CL, Moehring F, Kwan KY, Stucky CL. Sensory Neuron-Specific Deletion of TRPA1 Results in Mechanical Cutaneous Sensory Deficits. eNeuro. 2017;4(1):ENEURO.0069-16.2017.

35. Lennertz RC, Kossyreva EA, Smith AK, Stucky CL. TRPA1 mediates mechanical sensitization in nociceptors during inflammation. PLoS ONE. 2012;7(8):e43597.

36. Eid SR, et al. HC-030031, a TRPA1 selective antagonist, attenuates inflammatory- and neuropathy-induced mechanical hypersensitivity. Mol Pain. 2008;4:48.

37. Garrison SR, Stucky CL. Contribution of transient receptor potential ankyrin 1 to chronic pain in aged mice with complete Freund's adjuvant-induced arthritis. Arthritis Rheumatol. 2014;66(9):2380-2390.

38. Wei H, Hämäläinen MM, Saarnilehto M, Koivisto A, Pertovaara A. Attenuation of mechanical hypersensitivity by an antagonist of the TRPA1 ion channel in diabetic animals. Anesthesiology. 2009;111(1):147-154.

39. Moran MM, Szallasi A. Targeting nociceptive transient receptor potential channels to treat chronic pain: current state of the field [published online ahead of print September 19, 2017]. Br J Pharmacol. https://doi.org/10.1111/bph.14044

40. Walker KM, et al. The VR1 antagonist capsazepine reverses mechanical hyperalgesia in models of inflammatory and neuropathic pain. J Pharmacol Exp Ther. 2003;304(1):56-62.

41. Jones RC, Xu L, Gebhart GF. The mechanosensitivity of mouse colon afferent fibers and their sensitization by inflammatory mediators require transient receptor potential vanilloid 1 and acid-sensing ion channel 3. J Neurosci. 2005;25(47):10981-10989.

42. Hillery CA, et al. Transient receptor potential vanilloid 1 mediates pain in mice with severe sickle cell disease. Blood. 2011;118(12):3376-3383.

43. Honore P, et al. A-425619 [1-isoquinolin-5-yl-3-(4-trifluoromethyl-benzyl)-urea], a novel transient receptor potential type V1 receptor antagonist, relieves pathophysiological pain associated with inflammation and tissue injury in rats. J Pharmacol Exp Ther. 2005;314(1):410-421.

44. Caterina MJ, Schumacher MA, Tominaga M, Rosen TA, Levine JD, Julius D. The capsaicin receptor: a heat-activated ion channel in the pain pathway. Nature. 1997;389(6653):816-824

45. McNamara CR, et al. TRPA1 mediates formalin-induced pain. Proc Natl Acad Sci USA. 2007;104(33):13525-13530.

46. Meikle PJ, Hopwood JJ, Clague AE, Carey WF. Prevalence of lysosomal storage disorders. JAMA. 1999;281(3):249-254.

47. Spada M, et al. High incidence of later-onset fabry disease revealed by newborn screening. Am J Hum Genet. 2006;79(1):31-40.

48. Burton BK, et al. Newborn Screening for Lysosomal Storage Disorders in Illinois: The Initial 15-Month Experience. J Pediatr. 2017;190:130-135.

49. Elliott S, et al. Pilot study of newborn screening for six lysosomal storage diseases using Tandem Mass Spectrometry. Mol Genet Metab. 2016;118(4):304-309.

50. Eng CM, et al. A phase $1 / 2$ clinical trial of enzyme replacement in fabry disease: pharmacokinetic, substrate clearance, and safety studies. Am J Hum Genet. 2001;68(3):711-722.

51. Schiffmann R, et al. Enzyme replacement therapy in Fabry disease: a randomized controlled trial. JAMA. 2001;285(21):2743-2749.

52. Rombach SM, Hollak CE, Linthorst GE, Dijkgraaf MG. Cost-effectiveness of enzyme replacement therapy for Fabry disease. Orphanet J Rare Dis. 2013;8:29.

53. Barbey F, Livio F. Safety of enzyme replacement therapy. In: Mehta A, Beck M, Sunder-Plassmann G, eds. Fabry Disease: Perspectives from 5 Years of FOS. Oxford: Oxford PharmaGenesis; 2006. URL: https://www.ncbi.nlm.nih.gov/books/NBK11617/. Accessed February 27, 2018.

54. Tesmoingt C, et al. Enzyme therapy in Fabry disease: severe adverse events associated with anti-agalsidase cross-reactive IgG antibodies. Br J Clin Pharmacol. 2009;68(5):765-769.

55. Biegstraaten M, et al. Recommendations for initiation and cessation of enzyme replacement therapy in patients with Fabry disease: the European Fabry Working Group consensus document. Orphanet J Rare Dis. 2015;10:36.

56. Hopkin RJ, et al. Characterization of Fabry disease in 352 pediatric patients in the Fabry Registry. Pediatr Res. 2008;64(5):550-555

57. Eng CM, et al. Fabry disease: baseline medical characteristics of a cohort of 1765 males and females in the Fabry Registry. J Inherit Metab Dis. 2007;30(2):184-192.

58. Politei JM, et al. Pain in Fabry Disease: Practical Recommendations for Diagnosis and Treatment. CNS Neurosci Ther. 2016;22(7):568-576

59. Burlina AP, et al. Early diagnosis of peripheral nervous system involvement in Fabry disease and treatment of neuropathic pain the report of an expert panel. BMC Neurol. 2011;11:61.

60. Gold KF, et al. Quality of life of patients with Fabry disease. Qual Life Res. 2002;11(4):317-327.

61. Lakomá J, Rimondini R, Ferrer Montiel A, Donadio V, Liguori R, Caprini M. Increased expression of Trpv1 in peripheral terminals mediates thermal nociception in Fabry disease mouse model. Mol Pain. 2016;744806916663729.

62. Ziegler RJ, et al. Correction of the Biochemical and Functional Deficits in Fabry Mice Following AAV8-mediated Hepatic Expression of $\alpha$-galactosidase A. Mol Ther. 2007;15(3):492-500.

63. Namer B, et al. Changes in Ionic Conductance Signature of Nociceptive Neurons Underlying Fabry Disease Phenotype. Front Neurol. 2017;8:335.

64. Choi L, et al. The Fabry disease-associated lipid Lyso-Gb3 enhances voltage-gated calcium currents in sensory neurons and causes pain. Neurosci Lett. 2015;594:163-168. 
65. Anishkin A, Kung C. Stiffened lipid platforms at molecular force foci. Proc Natl Acad Sci USA. 2013;110(13):4886-4892.

66. Anishkin A, Loukin SH, Teng J, Kung C. Feeling the hidden mechanical forces in lipid bilayer is an original sense. Proc Natl Acad Sci USA. 2014;111(22):7898-7905.

67. Pliotas C, et al. The role of lipids in mechanosensation. Nat Struct Mol Biol. 2015;22(12):991-998.

68. Sághy É, et al. Evidence for the role of lipid rafts and sphingomyelin in Ca2+-gating of Transient Receptor Potential channels in trigeminal sensory neurons and peripheral nerve terminals. Pharmacol Res. 2015;100:101-116.

69. Jimenez-Andrade JM, Herrera MB, Ghilardi JR, Vardanyan M, Melemedjian OK, Mantyh PW. Vascularization of the dorsal root ganglia and peripheral nerve of the mouse: implications for chemical-induced peripheral sensory neuropathies. Mol Pain. 2008;4:10.

70. Godel T, et al. Human dorsal root ganglion in vivo morphometry and perfusion in Fabry painful neuropathy. Neurology. 2017;89(12):1274-1282.

71. Kaye EM, Kolodny EH, Logigian EL, Ullman MD. Nervous system involvement in Fabry's disease: clinicopathological and biochemical correlation. Ann Neurol. 1988;23(5):505-509.

72. Egan TJ, Acuna MA, Zenobi-Wong M, Zeilhofer HU, Urech D. Effects of N-Glycosylation of the human cation channel TRPA1 on agonist-sensitivity. Biosci Rep. 2016; 36(5): e00390.

73. Pertusa M, Madrid R, Morenilla-Palao C, Belmonte C, Viana F. N-glycosylation of TRPM8 ion channels modulates temperature sensitivity of cold thermoreceptor neurons. J Biol Chem. 2012;287(22):18218-18229.

74. Veldhuis NA, et al. N-glycosylation determines ionic permeability and desensitization of the TRPV1 capsaicin receptor. $J$ Biol Chem. 2012;287(26):21765-21772.

75. Cha SK, Ortega B, Kurosu H, Rosenblatt KP, Kuro-OM, Huang CL. Removal of sialic acid involving Klotho causes cell-surface retention of TRPV5 channel via binding to galectin-1. Proc Natl Acad Sci USA. 2008;105(28):9805-9810.

76. Jia Z, Ikeda R, Ling J, Viatchenko-Karpinski V, Gu JG. Regulation of Piezo2 Mechanotransduction by Static Plasma Membrane Tension in Primary Afferent Neurons. J Biol Chem. 2016;291(17):9087-9104.

77. Qi Y, Andolfi L, Frattini F, Mayer F, Lazzarino M, Hu J. Membrane stiffening by STOML3 facilitates mechanosensation in sensory neurons. Nat Commun. 2015;6:8512.

78. Cong L, et al. Multiplex genome engineering using CRISPR/Cas systems. Science. 2013;339(6121):819-823.

79. Geurts AM, et al. Generation of gene-specific mutated rats using zinc-finger nucleases. Methods Mol Biol. 2010;597:211-225.

80. Moreno C, Kennedy K, Andrae JW, Jacob HJ. Genome-wide scanning with SSLPs in the rat. Methods Mol Med. 2005;108:131-138

81. Desnick RJ, Allen KY, Desnick SJ, Raman MK, Bernlohr RW, Krivit W. Fabry's disease: enzymatic diagnosis of hemizygotes and heterozygotes. Alpha-galactosidase activities in plasma, serum, urine, and leukocytes. J Lab Clin Med. 1973;81(2):157-171

82. Sleat DE, Sohar I, Lackland H, Majercak J, Lobel P. Rat brain contains high levels of mannose-6-phosphorylated glycoproteins including lysosomal enzymes and palmitoyl-protein thioesterase, an enzyme implicated in infantile neuronal lipofuscinosis. J Biol Chem. 1996;271(32):19191-19198.

83. Doi K, et al. High-throughput screening identified disease-causing mutants and functional variants of $\alpha$-galactosidase A gene in Japanese male hemodialysis patients. J Hum Genet. 2012;57(9):575-579.

84. Varki A, et al. Symbol Nomenclature for Graphical Representations of Glycans. Glycobiology. 2015;25(12):1323-1324.

85. Schnaar RL. Isolation of glycosphingolipids. Meth Enzymol. 1994;230:348-370.

86. Schnaar RL, Mahoney JA, Swank-Hill P, Tiemeyer M, Needham LK. Receptors for gangliosides and related glycosphingolipids on central and peripheral nervous system cell membranes. Prog Brain Res. 1994;101:185-197.

87. Boccuto L, et al. A mutation in a ganglioside biosynthetic enzyme, ST3GAL5, results in salt \& pepper syndrome, a neurocutaneous disorder with altered glycolipid and glycoprotein glycosylation. Hum Mol Genet. 2014;23(2):418-433.

88. Mehta N, et al. Mass Spectrometric Quantification of N-Linked Glycans by Reference to Exogenous Standards. J Proteome Res. 2016;15(9):2969-2980.

89. Anumula KR, Taylor PB. A comprehensive procedure for preparation of partially methylated alditol acetates from glycoprotein carbohydrates. Anal Biochem. 1992;203(1):101-108.

90. Aoki K, Perlman M, Lim JM, Cantu R, Wells L, Tiemeyer M. Dynamic developmental elaboration of N-linked glycan complexity in the Drosophila melanogaster embryo. J Biol Chem. 2007;282(12):9127-9142.

91. Kumagai T, Katoh T, Nix DB, Tiemeyer M, Aoki K. In-gel $\beta$-elimination and aqueous-organic partition for improved O- and sulfoglycomics. Anal Chem. 2013;85(18):8692-8699.

92. Wells L, Hart GW, Athens Guidelines for the Publication of Glycomics Data. Glycomics: building upon proteomics to advance glycosciences. Mol Cell Proteomics. 2013;12(4):833-835.

93. York WS, et al. MIRAGE: the minimum information required for a glycomics experiment. Glycobiology. 2014;24(5):402-406.

94. Barabas ME, Kossyreva EA, Stucky CL. TRPA1 is functionally expressed primarily by IB4-binding, non-peptidergic mouse and rat sensory neurons. PLoS One. 2012;7(10):e47988.

95. Weyer AD, O'Hara CL, Stucky CL. Amplified Mechanically Gated Currents in Distinct Subsets of Myelinated Sensory Neurons following In Vivo Inflammation of Skin and Muscle. J Neurosci. 2015;35(25):9456-9462. 